TRANSACTIONS OF THE

AMERICAN MATHEMATICAL SOCIETY

Volume 354, Number 10, Pages 4179-4199

S 0002-9947(02)03058-1

Article electronically published on June 3, 2002

\title{
GENERALIZED PSEUDO-RIEMANNIAN GEOMETRY
}

\author{
MICHAEL KUNZINGER AND ROLAND STEINBAUER
}

\begin{abstract}
Generalized tensor analysis in the sense of Colombeau's construction is employed to introduce a nonlinear distributional pseudo-Riemannian geometry. In particular, after deriving several characterizations of invertibility in the algebra of generalized functions, we define the notions of generalized pseudo-Riemannian metric, generalized connection and generalized curvature tensor. We prove a "Fundamental Lemma of (pseudo-) Riemannian geometry" in this setting and define the notion of geodesics of a generalized metric. Finally, we present applications of the resulting theory to general relativity.
\end{abstract}

\section{INTRODUCTION}

Recently the theory of algebras of generalized functions (Colombeau algebras) [7], [8] has been restructured to allow for applications in a geometrical context [12, 13, 24]. The need for the latter has been clearly demonstrated by the use of nonlinear generalized function methods in the field of Lie group analysis of partial differential equations (e.g., 21], [9]) and the study of singular spacetimes in general relativity (see 40 for a survey). While diffeomorphism invariance in the so-called full version of Colombeau's construction (distinguished by a canonical embedding of the space of Schwartz distributions) for the scalar case was established in [12. 13. using calculus in infinite-dimensional (convenient, see [19]) vector spaces, the basic building blocks of the so-called special (or simplified) setting are a priori invariant under the action of a diffeomorphism. The latter, although not providing a distinguished embedding of distributions allows us to model singularities in a nonlinear context in a flexible and efficient way. A systematic development of global analysis using this framework has been started in [10, 24, 20]. In the present work we extend this approach by introducing the foundations of a generalized pseudoRiemannian geometry with special emphasis on applications in the theory of general relativity. For an alternative approach based on geometry of vector sheaves ([25]), see [26] 27].

We start with an in-depth discussion of the notion of invertibility in the algebra of generalized functions in Section 2 thereby setting the stage for the definition of a generalized pseudo-Riemannian metric on a manifold (Section 3). In Section 4 we extend the constructions of [20] by introducing the notion of a generalized section on a generalized mapping, which, in particular, allows us to define geodesics of a

Received by the editors August 9, 2001 and, in revised form, January 31, 2002.

2000 Mathematics Subject Classification. Primary 46F30; Secondary 46T30, 46F10, 83C05.

Key words and phrases. Algebras of generalized functions, Colombeau algebras, generalized tensor fields, generalized metric, (generalized) pseudo-Riemannian geometry, general relativity.

This work was in part supported by research grant P12023-MAT of the Austrian Science Fund. 
generalized metric. Section 5 is devoted to a systematic investigation of pseudoRiemannian geometry in this setting: in particular, we introduce generalized connections and prove a "Fundamental Lemma of (pseudo-) Riemannian geometry" as well as several consistency results with respect to (linear) distributional geometry, resp. the smooth setting. Finally in Section 6, after defining the relevant curvature quantities, we give a brief account on applications in general relativity. The remainder of the present section is devoted to a short review of Colombeau's construction (in its special variant) and, in particular, the global setting introduced in [24]. We begin by fixing some notation from differential geometry.

Throughout this paper $X$ and $Y$ denote paracompact, smooth Hausdorff manifolds of dimension $n$ resp. $m$. We denote vector bundles with base space $X$ by $\left(E, X, \pi_{X}\right)$ or simply by $E \rightarrow X$ and write a vector bundle chart $(V, \Psi)$ over a chart $(V, \psi)$ of the base $X$ in the form

$$
\begin{aligned}
\Psi: \pi^{-1}(V) & \rightarrow \psi(V) \times \mathbb{K}^{n^{\prime}} \\
z & \mapsto\left(\psi(p), \psi^{1}(z), \ldots, \psi^{n^{\prime}}(z)\right) \equiv(\psi(p), \boldsymbol{\psi}(z))
\end{aligned}
$$

where $p=\pi(z)$ and $\mathbb{K}^{n^{\prime}}$ (with $\mathbb{K}=\mathbb{R}$ or $\mathbb{K}=\mathbb{C}$ ) is the typical fiber. Given a vector bundle atlas $\left(V_{\alpha}, \Psi_{\alpha}\right)_{\alpha}$, we write the change of chart in the form $\Psi_{\alpha} \circ \Psi_{\beta}(y, w)=$ $\left(\psi_{\alpha \beta}(y), \boldsymbol{\psi}_{\alpha \beta}(y) w\right)$, where $\psi_{\alpha \beta}:=\psi_{\alpha} \circ \psi_{\beta}^{-1}$ and $\psi_{\alpha \beta}: \psi_{\beta}\left(V_{\alpha} \cap V_{\beta}\right) \rightarrow \operatorname{GL}\left(n^{\prime}, \mathbb{K}\right)$ denotes the transition functions.

For vector bundles $E \rightarrow X$ and $F \rightarrow Y$, we denote the space of vector bundle homomorphisms from $E$ to $F$ by $\operatorname{Hom}(E, F)$. Given $f \in \operatorname{Hom}(E, F)$ the induced smooth map on the bases is denoted by $\underline{f}$, i.e., $\pi_{Y} \circ f=\underline{f} \circ \pi_{X}$. For vector bundle charts $(V, \Phi)$ of $E$ and $(W, \Psi)$ of $F$ we write the local vector bundle homomorphism $f_{\Psi \Phi}:=\Psi \circ f \circ \Phi^{-1}: \varphi\left(V \cap \underline{f}^{-1}(W)\right) \times \mathbb{K}^{n^{\prime}} \rightarrow \phi(W) \times \mathbb{K}^{m^{\prime}}$ in the form

$$
f_{\Psi \Phi}(x, \xi)=\left(f_{\Psi \Phi}^{(1)}(x), f_{\Psi \Phi}^{(2)}(x) \cdot \xi\right)
$$

The space of $\mathcal{C}^{k}$-sections of a vector bundle $E \rightarrow X$ is denoted by $\Gamma^{k}(X, E)$, and we drop the superscript in case $k=\infty$. The $(r, s)$-tensor bundle over $X$ will be denoted by $T_{s}^{r}(X)$, and we use the following notation for spaces of tensor fields $\mathcal{T}_{s}^{r}(X):=\Gamma\left(X, T_{s}^{r}(X)\right), \mathfrak{X}:=\Gamma(X, T X)$ and $\mathfrak{X}^{*}:=\Gamma\left(X, T^{*} X\right)$, where $T X$ and $T^{*} X$ denote the tangent and cotangent bundle of $X$, respectively. For a section $s \in \Gamma(X, E)$ we call $s_{\alpha}^{i}=\Psi_{\alpha}^{i} \circ s \circ \psi_{\alpha}^{-1}$ its $i$-th component $\left(1 \leq i \leq n^{\prime}\right)$ with respect to the vector bundle chart $\left(V_{\alpha}, \Psi_{\alpha}\right)$.

The space of $E$-valued distributions of density character $q$ (see, e.g., [37], Chap. 2) will be denoted by $\mathcal{D}^{\prime}\left(X, E \otimes \operatorname{Vol}^{q}(X)\right)$ (where $\operatorname{Vol}^{q}(X)$ is the $q$-volume bundle of $X)$; in particular, the space of $(r, s)$-tensor distributions $\left(q=0\right.$ and $\left.E=T_{s}^{r}(X)\right)$ will be denoted by $\mathcal{D}_{s}^{\prime r}(X)$. Whenever convenient we shall use summation convention and abstract index notation (cf. 35, Chap. 2). That is, we denote an $(r, s)$-tensor field by $T_{b_{1} \ldots b_{s}}^{a_{1} \ldots a_{r}} \in \mathcal{T}_{s}^{r}(X)$ while Greek indices, i.e., $T_{\beta_{1} \ldots \beta_{s}}^{\alpha_{1} \ldots \alpha_{r}}$, are used to denote its components with respect to a certain basis. Hence equations involving Latin indices are "true" tensor equations holding in any basis.

The (special) algebra of generalized functions on $X$ is defined as the quotient $\mathcal{G}(X):=\mathcal{E}_{M}(X) / \mathcal{N}(X)$ of the space $\mathcal{E}_{M}(X)$ of nets of smooth functions $\left(u_{\varepsilon}\right)_{\varepsilon \in(0,1]} \in$ $\mathcal{C}^{\infty}(X)^{(0,1]}=: \mathcal{E}(X)$ of moderate growth modulo the space $\mathcal{N}(X)$ of negligible nets, where the respective notions of moderateness and negligibility are defined (denoting by $\mathcal{P}(X)$ the space of linear differential operators on $X$ ) by the following asymptotic 
estimates

$$
\begin{aligned}
& \mathcal{E}_{M}(X):=\left\{\left(u_{\varepsilon}\right)_{\varepsilon} \in \mathcal{E}(X): \forall K \subset \subset X, \forall P \in \mathcal{P}(X) \exists N \in \mathbb{N}:\right.\left.\sup _{p \in K}\left|P u_{\varepsilon}(p)\right|=O\left(\varepsilon^{-N}\right)\right\}, \\
&\left.\mathcal{N}(X):=\left\{\left(u_{\varepsilon}\right)_{\varepsilon} \in \mathcal{E}_{M}(X): \forall K \subset \subset X, \forall m \in \mathbb{N}_{0}: \sup _{p \in K}\left|u_{\varepsilon}(p)\right|=O\left(\varepsilon^{m}\right)\right)\right\} .
\end{aligned}
$$

Elements of $\mathcal{G}(X)$ are denoted by $u=\operatorname{cl}\left[\left(u_{\varepsilon}\right)_{\varepsilon}\right]=\left(u_{\varepsilon}\right)_{\varepsilon}+\mathcal{N}(X) \cdot \mathcal{G}\left({ }_{-}\right)$is a fine sheaf of differential algebras with respect to the Lie derivative (w.r.t. smooth vector fields) defined by $L_{\xi} u:=\operatorname{cl}\left[\left(L_{\xi} u_{\varepsilon}\right)_{\varepsilon}\right]$.

The spaces of moderate resp. negligible sequences and hence the algebra itself may be characterized locally, i.e., $u \in \mathcal{G}(X)$ if and only if $u_{\alpha}:=u \circ \psi_{\alpha}^{-1} \in \mathcal{G}\left(\psi_{\alpha}\left(V_{\alpha}\right)\right)$ for all charts $\left(V_{\alpha}, \psi_{\alpha}\right)$ and $\left.u_{\alpha}\right|_{\psi_{\alpha}\left(V_{\alpha} \cap V_{\beta}\right)}=\left.u_{\beta}\right|_{\psi_{\beta}\left(V_{\alpha} \cap V_{\beta}\right)} \circ \psi_{\beta} \circ \psi_{\alpha}^{-1}$ for all $\alpha, \beta$ with $V_{\alpha} \cap V_{\beta} \neq \emptyset$.

Smooth functions are embedded into $\mathcal{G}$ simply by the "constant" embedding $\sigma$, i.e., $\sigma(f):=\operatorname{cl}\left[(f)_{\varepsilon}\right]$; hence $\mathcal{C}^{\infty}(X)$ is a subalgebra of $\mathcal{G}(X)$. Moreover, there exist injective sheaf morphisms $\iota: \mathcal{D}^{\prime}\left(_{-}\right) \hookrightarrow \mathcal{G}\left({ }_{-}\right)$which coincide with $\sigma$ on $\mathcal{C}^{\infty}\left(_{-}\right)$ (24] Th. 2). These, however, are not canonical (cf. the discussion in [24], Sec. 4). In fact, such embeddings depend (in addition to the choice of a mollifier as in the $\mathbb{R}^{n}$-case) also on the choice of an atlas for $X$ and of families of cut-off functions, hence are non-geometric in an essential way. Nevertheless, there are a number of physically relevant cases (examples will be given in Section 6) where a distinguished regularization procedure inducing an embedding into the algebra is suggested by the application under consideration. Additionally it is often possible to show independence of the distributional results achieved (via the concept of association) through such a procedure from the choice of embedding. In cases where a canonical embedding is required, on the other hand, it is possible to employ the intrinsic full Colombeau algebra (providing a canonical embedding of $\mathcal{D}^{\prime}$ ), see 13. A theory of generalized pseudo-Riemannian geometry in this full setting is the subject of ongoing research.

Compatibility with respect to the distributional setting is established via the notion of association, defined as follows: a generalized function $u$ is called associated with $0, u \approx 0$, if $\int_{X} u_{\varepsilon} \mu \rightarrow 0(\varepsilon \rightarrow 0)$ for all compactly supported one-densities $\mu$ and one (hence every) representative $\left(u_{\varepsilon}\right)_{\varepsilon}$ of $u$. Clearly, $\approx$ induces an equivalence relation giving rise to a linear quotient space of $\mathcal{G}(X)$, which generalizes the notion of distributional equality to the level of the algebra. If $\int_{X} u_{\varepsilon} \mu \rightarrow\langle w, \mu\rangle$ for some $w \in \mathcal{D}^{\prime}(X)$ (where $\langle$,$\rangle denotes the distributional action), then w$ is called the distributional shadow (or macroscopic aspect) of $u$ and we write $u \approx w$. If it exists at all, the latter is unique. In the absence of a distinguished embedding it is useful to define also the stronger notion of $k$-association. We call a generalized function $u k$-associated with $0(0 \leq k \leq \infty), u \approx_{k} 0$, if for all $l \leq k$, all $\xi_{1}, \ldots, \xi_{l} \in \mathfrak{X}(X)$ and one (hence every) representative $L_{\xi_{1}} \ldots L_{\xi_{l}} u_{\varepsilon} \rightarrow 0$ uniformly on compact sets. Also we say that $u$ admits $f$ as a $\mathcal{C}^{k}$-associated function, $u \approx_{k} f$, if for all $l \leq k$, all $\xi_{1}, \ldots, \xi_{l} \in \mathfrak{X}(X)$ and one (hence any) representative $L_{\xi_{1}} \ldots L_{\xi_{l}}\left(u_{\varepsilon}-f\right) \rightarrow 0$ uniformly on compact sets. The concept of $k$-association provides a close connection to the classical $\left(\mathcal{C}^{k}\right)$ picture: when modelling singular data in applications it is often possible to obtain $\mathcal{C}^{k}$-associated functions which serve to establish consistency properties with the classical setting (cf. Proposition 5.4 or Theorem 6.2 below). 
Finally, inserting $p \in X$ into $u \in \mathcal{G}(X)$ yields a well-defined element of the ring of constants $\mathcal{K}$ (corresponding to $\mathbb{K}=\mathbb{R}$ resp. $\mathbb{C}$ ), defined as the set of moderate nets of numbers $\left(\left(r_{\varepsilon}\right)_{\varepsilon} \in \mathbb{K}^{(0,1]}\right.$ with $\left|r_{\varepsilon}\right|=O\left(\varepsilon^{-N}\right)$ for some $\left.N\right)$ modulo negligible nets $\left(\left|r_{\varepsilon}\right|=O\left(\varepsilon^{m}\right)\right.$ for each $\left.m\right)$. Moreover, generalized functions on $X$ are characterized by their generalized point values - a feature which distinguishes them from the purely distributional setting. On $X$ define the space

$$
\widetilde{X}_{c}:=X_{c} / \sim
$$

of equivalence classes of compactly supported nets $\left(p_{\varepsilon}\right)_{\varepsilon} \in X^{(0,1]}$ with respect to the equivalence relation $p_{\varepsilon} \sim p_{\varepsilon}^{\prime}: \Leftrightarrow d_{h}\left(p_{\varepsilon}, p_{\varepsilon}^{\prime}\right)=O\left(\varepsilon^{m}\right)$ for all $m$, where $d_{h}$ denotes the distance function on $X$ induced by any Riemannian metric. Then for any generalized function $u$ and any $\tilde{p} \in \widetilde{X}_{c}$, the insertion $u(\tilde{p})$ yields a well-defined element of $\mathcal{K}$ and $u=0 \in \mathcal{G}(X)$ if and only if $u(\tilde{p})=0 \in \mathcal{K}$ for all generalized points $\tilde{p} \in \widetilde{X}_{c}(24]$, Th. 1).

The $\mathcal{G}(X)$-module of generalized sections $\Gamma_{\mathcal{G}}(X, E)$ of a vector bundle $E \rightarrow X$ is defined along the same lines using analogous asymptotic estimates with respect to the norm induced by any Riemannian metric on the respective fibers. More precisely, setting $\Gamma_{\mathcal{E}}(X, E):=(\Gamma(X, E))^{(0,1]}$ we define $(\mathcal{P}(X, E)$ denoting the space of linear differential operators on $\Gamma(X, E))$

$$
\begin{aligned}
& \Gamma_{\mathcal{E}_{M}}(X, E):=\left\{\left(s_{\varepsilon}\right)_{\varepsilon} \in \Gamma_{\mathcal{E}}(X, E):\right. \forall P \in \mathcal{P}(X, E) \forall K \subset \subset X \exists N \in \mathbb{N}: \\
&\left.\sup _{p \in K}\left\|P u_{\varepsilon}(p)\right\|=O\left(\varepsilon^{-N}\right)\right\}, \\
& \Gamma_{\mathcal{N}}(X, E):=\left\{\left(s_{\varepsilon}\right)_{\varepsilon} \in \Gamma_{\mathcal{E}_{M}}(X, E):\right. \forall K \subset \subset X \forall m \in \mathbb{N}: \\
&\left.\sup _{p \in K}\left\|u_{\varepsilon}(p)\right\|=O\left(\varepsilon^{m}\right)\right\},
\end{aligned}
$$

and finally,

$$
\Gamma_{\mathcal{G}}(X, E):=\Gamma_{\mathcal{E}_{M}}(X, E) / \Gamma_{\mathcal{N}}(X, E) .
$$

We denote generalized sections by $s=\operatorname{cl}\left[\left(s_{\varepsilon}\right)_{\varepsilon}\right]=\left(s_{\varepsilon}\right)_{\varepsilon}+\mathcal{N}(X, E)$. Alternatively we may describe a section $s \in \Gamma_{\mathcal{G}}(X, E)$ by a family $\left(s_{\alpha}\right)_{\alpha}=\left(\left(s_{\alpha}^{i}\right)_{\alpha}\right)_{i=1}^{n^{\prime}}$, where $s_{\alpha}$ is called the local expression of $s$ with its components $s_{\alpha}^{i}:=\Psi_{\alpha}^{i} \circ s \circ \psi_{\alpha}^{-1} \in \mathcal{G}\left(\psi_{\alpha}\left(V_{\alpha}\right)\right)$ $\left(i=1, \ldots, n^{\prime}\right)$ satisfying $s_{\alpha}^{i}(x)=\left(\boldsymbol{\psi}_{\alpha \beta}\right)_{j}^{i}\left(\psi_{\beta} \circ \psi_{\alpha}^{-1}(x)\right) s_{\beta}^{j}\left(\psi_{\beta} \circ \psi_{\alpha}^{-1}(x)\right)$ for all $x \in \psi_{\alpha}\left(V_{\alpha} \cap V_{\beta}\right)$, where $\psi_{\alpha \beta}$ denotes the transition functions of the bundle. Smooth sections of $E \rightarrow X$ again may be embedded as constant nets, i.e., we define an embedding $\Sigma: \Gamma(X, E) \hookrightarrow \Gamma_{\mathcal{G}}(X, E)$ by $\Sigma(s)=\operatorname{cl}\left[(s)_{\varepsilon}\right]$.

$\Gamma_{\mathcal{G}}(-, E)$ is a fine sheaf of $\mathcal{G}(X)$-modules. Moreover, the $\mathcal{G}(X)$-module $\mathcal{G}(X, E)$ is projective and finitely generated $\left([24\right.$, Th. 5$)$. Since $\mathcal{C}^{\infty}(X)$ is a subring of $\mathcal{G}(X)$, $\Gamma_{\mathcal{G}}(X, E)$ also may be viewed as a $\mathcal{C}^{\infty}(X)$-module and the two respective module structures are compatible with respect to the embeddings. Furthermore, we have the following algebraic characterization of the space of generalized sections ([24, Th. 4):

$$
\Gamma_{\mathcal{G}}(X, E)=\mathcal{G}(X) \otimes \Gamma(X, E),
$$

where the tensor product is taken over the module $\mathcal{C}^{\infty}(X)$.

Compatibility with respect to the classical resp. distributional setting again is accomplished using the concept of ( $k$-)association. A section $s \in \Gamma_{\mathcal{G}}(X, E)$ is called associated with $0, s \approx 0$, if all its components $s_{\alpha}^{i} \approx 0$ in $\mathcal{G}\left(\psi_{\alpha}\left(V_{\alpha}\right)\right)$. $s$ allows for the 
distributional shadow $w, s \approx w \in \mathcal{D}^{\prime}(X, E)$, if $s_{\alpha}^{i} \approx w_{\alpha}^{i} \in \mathcal{G}\left(\psi_{\alpha}\left(V_{\alpha}\right)\right)$ for all $\alpha, i$ (and $w_{\alpha}^{i}$ denoting the local expression of the distribution $w$ ). Similarly $s$ is called $\mathcal{C}^{k}$ associated with $0(0 \leq k \leq \infty), s \approx_{k} 0$, if for one (hence every) representative $\left(s_{\varepsilon}\right)_{\varepsilon}$, all components $s_{\alpha \varepsilon}^{i} \rightarrow 0$ uniformly on compact sets in all derivatives of order less than or equal to $k$. We say that $s$ allows $t \in \Gamma^{k}(X, E)$ as a $\mathcal{C}^{k}$-associated section, $s \approx_{k} t$, if for one (hence every) representative $\left(s_{\varepsilon}\right)_{\varepsilon}$, all components $s_{\alpha \varepsilon}^{i} \rightarrow t_{\alpha}^{i}$ uniformly on compact sets in all derivatives of order less than or equal to $k$.

Generalized tensor fields (i.e., elements of $\mathcal{G}_{s}^{r}(X):=\Gamma_{\mathcal{G}}\left(X, T_{s}^{r}(X)\right)$ ) may be viewed likewise as $\mathcal{C}^{\infty}(X)$-multilinear mappings taking smooth vector fields resp. one-forms to $\mathcal{G}(X)$ or as $\mathcal{G}(X)$-multilinear mappings taking generalized vector resp. covector fields to generalized functions, i.e., as $\mathcal{C}^{\infty}(X)$-resp. $\mathcal{G}(X)$-modules we have (24], Th. 6)

$$
\begin{aligned}
& \mathcal{G}_{s}^{r}(X) \cong L_{\mathcal{C}^{\infty}(X)}\left(\mathfrak{X}^{*}(X)^{r}, \mathfrak{X}(X)^{s} ; \mathcal{G}(X)\right), \\
& \mathcal{G}_{s}^{r}(X) \cong L_{\mathcal{G}(X)}\left(\mathcal{G}_{1}^{0}(X)^{r}, \mathcal{G}_{0}^{1}(X)^{s} ; \mathcal{G}(X)\right) .
\end{aligned}
$$

Given a generalized tensor field $T \in \mathcal{G}_{s}^{r}(X)$, we shall call the $n^{r+s}$ generalized functions on $V_{\alpha}$ defined by

$$
T_{\gamma_{1} \ldots \gamma_{s}}^{\alpha \beta_{1} \ldots \beta_{r}}:=\left.T\right|_{V_{\alpha}}\left(d x^{\beta_{1}}, \ldots, d x^{\beta_{r}}, \partial_{\gamma_{1}}, \ldots, \partial_{\gamma_{s}}\right)
$$

its components with respect to the chart $\left(V_{\alpha}, \psi_{\alpha}\right)$.

In 24] many concepts of classical differential geometry (in particular, Lie derivatives with respect to both smooth and generalized vector fields, Lie brackets, tensor product, contraction, exterior algebra, etc.) have been generalized to this new setting and will be used in the sequel.

\section{INVERTIBILITY}

Prior to our analysis of generalized semi-Riemannian geometry, in the present section we are going to derive several characterization results concerning invertibility in the Colombeau algebra which will be essential for the algebraic aspects of the theory to be developed in the subsequent sections.

We begin with a characterization of multiplicative invertibility in $\mathcal{G}(X)$.

Proposition 2.1. Let $u \in \mathcal{G}(X)$. The following are equivalent.

(i) There exists $v \in \mathcal{G}(X)$ with $u v=1$.

(ii) For each representative $\left(u_{\varepsilon}\right)_{\varepsilon}$ of $u$ and each $K \subset \subset X$ there exist $\varepsilon_{0}>0$ and $q \in \mathbb{N}$ such that $\inf _{p \in K}\left|u_{\varepsilon}(p)\right| \geq \varepsilon^{q}$ for all $\varepsilon<\varepsilon_{0}$.

Proof. (i) $\Rightarrow$ (ii): Let $u=\operatorname{cl}\left[\left(u_{\varepsilon}\right)_{\varepsilon}\right]$ and $v=\operatorname{cl}\left[\left(v_{\varepsilon}\right)_{\varepsilon}\right]$. By assumption there exists $n=\operatorname{cl}\left[\left(n_{\varepsilon}\right)_{\varepsilon}\right] \in \mathcal{N}(X)$ such that $u_{\varepsilon} v_{\varepsilon} \equiv 1+n_{\varepsilon}$. We first claim that there exists some $\varepsilon_{0}$ such that $v_{\varepsilon}(p) \neq 0$ for all $p \in K$ and all $\varepsilon<\varepsilon_{0}$. Indeed, otherwise there would exist a zero-sequence $\varepsilon_{m}$ and a sequence $p_{m}$ in $K$ such that $v_{\varepsilon_{m}}\left(p_{m}\right)=0$ for all $m$. But then $0=u_{\varepsilon_{m}}\left(p_{m}\right) v_{\varepsilon_{m}}\left(p_{m}\right)=1+n_{\varepsilon_{m}}\left(p_{m}\right) \rightarrow 1(m \rightarrow \infty)$, a contradiction. Since $\left(v_{\varepsilon}\right)_{\varepsilon} \in \mathcal{E}_{M}(X)$ there exists $l \in \mathbb{N}$ and $\varepsilon_{1}>0$ with $\sup _{p \in K}\left|\left(v_{\varepsilon}\right)_{\varepsilon}(p)\right|<\varepsilon^{-l}$ for $\varepsilon<\varepsilon_{1}$. Hence

$$
\left|u_{\varepsilon}(p)\right|>\varepsilon^{l}\left(1-\left|n_{\varepsilon}(p)\right|\right)>\varepsilon^{l+1}
$$

for $\varepsilon$ small, uniformly for $p \in K$.

(ii) $\Rightarrow$ (i): Let $\left(X_{m}\right)_{m \in \mathbb{N}}$ be an open covering of $X$ consisting of relatively compact sets. Then by assumption, $v_{\varepsilon}^{m}(p):=\frac{1}{u_{\varepsilon}(p)}$ exists for $p \in X_{m}$ and $\varepsilon$ sufficiently small (for all other $\varepsilon$ we may set $v_{\varepsilon}^{m} \equiv 0$ ). Also, $\left(v_{\varepsilon}^{m}\right)_{\varepsilon} \in \mathcal{E}_{M}\left(X_{m}\right)$; so 
$v^{m}:=\operatorname{cl}\left[\left(v_{\varepsilon}^{m}\right)_{\varepsilon}\right]$ is a well-defined element of $\mathcal{G}\left(X_{m}\right)$. By definition, for $X_{m} \cap X_{k} \neq \emptyset$ we have $\left.v^{m}\right|_{X_{m} \cap X_{k}}=\left.v^{k}\right|_{X_{m} \cap X_{k}}$; so $\left\{v^{m} \mid m \in \mathbb{N}\right\}$ forms a coherent family. Since $\mathcal{G}(-)$ is a sheaf of differential algebras (cf. [10, 24]) it follows that there exists a unique element $v \in \mathcal{G}(X)$ with $\left.v\right|_{X_{m}}=v_{m}$ for all $m \in \mathbb{N}$. It is clear that $v$ is the desired multiplicative inverse of $u$.

We call an element $r \in \mathcal{K}$ strictly nonzero if there exists some representative $\left(r_{\varepsilon}\right)_{\varepsilon}$ and an $m \in \mathbb{N}$ with $\left|r_{\varepsilon}\right| \geq \varepsilon^{m}$ for $\varepsilon$ sufficiently small. By specializing 2.1 to $K=\{p\}(p \in X)$ it follows that $r$ is invertible if and only if it is strictly nonzero. Also, Proposition 2.1 can be restated as follows: a Colombeau function possesses a multiplicative inverse if and only if it is strictly nonzero, uniformly on compact sets.

Proposition 2.2. Let $r \in \mathcal{K}$. The following are equivalent:

(i) $r$ is not invertible;

(ii) $r$ is a zero divisor.

Proof. (i) $\Rightarrow$ (ii): By the above we have: $\forall N \in \mathbb{N} \forall \varepsilon_{0} \exists \varepsilon<\varepsilon_{0}$ : $\left|r_{\varepsilon}\right|<\varepsilon^{N}$. Thus there exists a sequence $\varepsilon_{k} \searrow 0$ with $\left|r_{\varepsilon_{k}}\right|<\varepsilon_{k}^{k}$. Define $\left(a_{\varepsilon}\right)_{\varepsilon}$ by $a_{\varepsilon}=r_{\varepsilon_{k+1}}$ for $\varepsilon_{k+1} \leq \varepsilon<\varepsilon_{k}$. Then

$$
\left|a_{\varepsilon}\right|=\left|r_{\varepsilon_{k+1}}\right|<\varepsilon_{k+1}^{k+1} \leq \varepsilon^{k+1} \quad\left(\varepsilon_{k+1} \leq \varepsilon<\varepsilon_{k}\right) .
$$

Hence $\left|a_{\varepsilon}\right|<\varepsilon^{N+1}$ for $0<\varepsilon<\varepsilon_{N}$, i.e., $\left(a_{\varepsilon}\right)_{\varepsilon} \in \mathcal{N}$. Setting $\tilde{r}_{\varepsilon}=r_{\varepsilon}-a_{\varepsilon}$ we obtain a representative $\tilde{r}$ of $r$ with $\tilde{r}_{\varepsilon_{k}}=0$ for all $k \in \mathbb{N}$. Denote by $s$ the class of $\left(s_{\varepsilon}\right)_{\varepsilon}$, where

$$
s_{\varepsilon}= \begin{cases}1, & \varepsilon \in\left\{\varepsilon_{1}, \varepsilon_{2}, \ldots\right\} \\ 0, & \varepsilon \notin\left\{\varepsilon_{1}, \varepsilon_{2}, \ldots\right\}\end{cases}
$$

Then $\tilde{r}_{\varepsilon} s_{\varepsilon} \equiv 0$, and so $r s=0$, but $s \neq 0$ in $\mathcal{K}$.

(ii) $\Rightarrow$ (i) is obvious.

A comprehensive study of algebraic properties of $\mathcal{K}$ can be found in [1]. Clearly the analogue of Proposition 2.2 is wrong for $\mathcal{C}^{\infty}(X)$. It is also false for $\mathcal{G}(X)$ :

Example 2.3. Let $X=\mathbb{R}$ and let $x$ denote the identical function on $\mathbb{R}$, considered as an element of $\mathcal{G}(\mathbb{R})$. Then $x$ is not invertible by Proposition 2.1 and we are going to show that it is not a zero divisor in $\mathcal{G}(\mathbb{R})$. To this end, let $u=\operatorname{cl}\left[\left(u_{\varepsilon}\right)_{\varepsilon}\right] \in \mathcal{G}(\mathbb{R})$ be such that $x u=0$ in $\mathcal{G}(\mathbb{R})$. Suppose that $\left(u_{\varepsilon}\right)_{\varepsilon} \notin \mathcal{N}(\mathbb{R})$. Since $\left(u_{\varepsilon}\right)_{\varepsilon}$ necessarily satisfies the $\mathcal{N}$-estimates on every compact set not containing 0 , it follows that there exists $K \subset \subset \mathbb{R}$ with $0 \in K, q_{0} \in \mathbb{N}, \varepsilon_{m} \searrow 0$ and $x_{m} \in K$ such that $\left|u_{\varepsilon_{m}}\left(x_{m}\right)\right|>\varepsilon_{m}^{q_{0}}$ for all $m \in \mathbb{N}$. Without loss of generality we may suppose that $x_{m} \rightarrow 0$. For any $r \in \mathbb{N}$ and $m$ large we have $\left|x_{m} u_{\varepsilon_{m}}\left(x_{m}\right)\right| \leq \varepsilon_{m}^{r}$. Hence $\left|x_{m}\right| \leq \varepsilon_{m}^{r-q_{0}}$ for these $m$, i.e., $x_{m}$ goes to 0 faster than any power of $\varepsilon$. Now

$$
\left|u_{\varepsilon_{m}}\left(x_{m}\right)\right| \leq\left|x_{m} u_{\varepsilon_{m}}^{\prime}\left(x_{m}\right)\right|+\left|\left(x u_{\varepsilon_{m}}\right)^{\prime}\left(x_{m}\right)\right| .
$$

Since $\left(u_{\varepsilon}\right)_{\varepsilon}$ is moderate there exists $N \in \mathbb{N}$ such that $\left|u_{\varepsilon_{m}}^{\prime}\left(x_{m}\right)\right| \leq \varepsilon_{m}^{-N}$. By the above, $\left|x_{m}\right| \leq \varepsilon_{m}^{N+q_{0}+1}$ for $m$ large and so, taking into account the $\mathcal{N}$-estimate for $\left(x u_{\varepsilon}\right)^{\prime}$ we obtain

$$
\left|u_{\varepsilon_{m}}\left(x_{m}\right)\right| \leq \varepsilon_{m}^{q_{0}+1} \quad(m \text { large })
$$

Consequently, $\varepsilon_{m}^{q_{0}} \leq \varepsilon_{m}^{q_{0}+1}$ for large $m$, a contradiction. 
We note that the notion of zero divisor in $\mathcal{C}^{\infty}(X)$ and $\mathcal{G}(X)$ differs: in fact (with $X=\mathbb{R}) e^{-1 / x^{2}}$ is obviously not a zero divisor in $\mathcal{C}^{\infty}(\mathbb{R})$ but $e^{-1 / x^{2}} \cdot \delta(x)=0$ in $\mathcal{G}(\mathbb{R})$; so it is a zero divisor in $\mathcal{G}(X)$.

An element $f$ of $\mathcal{C}^{\infty}(X)$ is invertible if and only if $f(p)$ is invertible in $\mathbb{R}$ for each $p \in X$. Our next aim is to find the appropriate generalization of this observation to the context of Colombeau algebras. A straightforward adaptation of the smooth case is impossible as is demonstrated by the following example:

Example 2.4. Let $X=\mathbb{R}$ and set

$$
u_{\varepsilon}(x):=\varepsilon^{\frac{x^{2}}{x^{4}+\varepsilon^{4}}} .
$$

The net $\left(u_{\varepsilon}\right)_{\varepsilon}$ is moderate since $\left|u_{\varepsilon}(x)\right| \leq 1$ and $u_{\varepsilon}^{(k)}(x)=r_{k}(x, \varepsilon, \ln (\varepsilon)) u_{\varepsilon}(x)$ with $r_{k}$ a rational function $(k \in \mathbb{N})$. Thus $u:=\operatorname{cl}\left[\left(u_{\varepsilon}\right)_{\varepsilon}\right]$ is a well-defined element of $\mathcal{G}(\mathbb{R})$. We are going to show that $u\left(x_{0}\right)$ is invertible in $\mathcal{K}$ for each $x_{0} \in \mathbb{R}$ but that $u$ is not invertible in $\mathcal{G}(\mathbb{R})$.

In fact, $u_{\varepsilon}(0)=1$ for all $\varepsilon$ and for $x_{0} \neq 0$ we have $u_{\varepsilon}\left(x_{0}\right) \geq \varepsilon^{\frac{1}{x_{0}^{2}}}$; so $u\left(x_{0}\right)$ is strictly nonzero, hence invertible in $\mathcal{K}$ for each $x_{0} \in \mathbb{R}$. However, $u$ is not uniformly strictly nonzero on compact sets, hence not invertible in $\mathcal{G}(\mathbb{R})$ by Proposition 2.1 To see this, take $K=[0,1]$. Then $x=\varepsilon \in K$, but $u_{\varepsilon}(\varepsilon)=\varepsilon^{\frac{1}{2 \varepsilon^{2}}} \geq \varepsilon^{q}$ cannot hold identically in $\varepsilon$ in any interval $\left(0, \varepsilon_{0}\right)$ for any $q \in \mathbb{N}$.

The correct generalization of the result in the smooth case uses the point value characterization of elements of $\mathcal{G}(X)$ derived in [32, [24]:

Proposition 2.5. $u \in \mathcal{G}(X)$ is invertible if and only if $u(\tilde{p})$ is invertible in $\mathcal{K}$ for each $\tilde{p} \in \widetilde{X}_{c}$.

Proof. The condition is obviously necessary. Conversely, suppose that $u \in \mathcal{G}(X)$ is not invertible. Then by Proposition 2.1 there exist $K \subset \subset X$ and sequences $\varepsilon_{m} \rightarrow 0$, $q_{m} \rightarrow \infty$ and $p_{m} \in K$ such that

$$
\left|u_{\varepsilon_{m}}\left(p_{m}\right)\right|<\varepsilon_{m}^{q_{m}} \quad(m \in \mathbb{N}) .
$$

Set $p_{\varepsilon}:=p_{m}$ for $\frac{1}{m+1}<\varepsilon \leq \frac{1}{m}$. Then $\tilde{p}:=\operatorname{cl}\left[\left(p_{\varepsilon}\right)_{\varepsilon}\right]$ defines a compactly supported generalized point in $X$, i.e., an element of $\widetilde{X}_{c}$. By construction, $u(\tilde{p})$ is not invertible in $\mathcal{K}$.

Finally, we shall need the following characterization of nondegeneracy in $\mathcal{K}^{n}$ :

Lemma 2.6. Let $A \in \mathcal{K}^{n^{2}}$. The following are equivalent:

(i) $A$ is nondegenerate, i.e., $\xi \in \mathcal{K}^{n}, \xi^{t} A \eta=0 \forall \eta \in \mathcal{K}^{n}$ implies $\xi=0$;

(ii) $A: \mathcal{K}^{n} \rightarrow \mathcal{K}^{n}$ is injective;

(iii) $A: \mathcal{K}^{n} \rightarrow \mathcal{K}^{n}$ is bijective;

(iv) $\operatorname{det}(A)$ is invertible.

Proof. (ii) $\Leftrightarrow$ (iii) $\Leftrightarrow$ (iv): By [5], Ch. III, $\S 8$, Prop. 3, (ii) is equivalent with $\operatorname{det}(A)$ not being a zero divisor in $\mathcal{K}$. [5], Ch. III, $\S 8$, Th. 1 shows that (iii) is equivalent with $\operatorname{det}(A)$ being invertible. Hence the claim follows from Proposition 2.2.

(i) $\Leftrightarrow$ (ii): First (i) is equivalent with $A^{t}$ being injective. Indeed $\xi^{t} A \eta=0 \forall \eta \in$ $\mathcal{K}^{n} \Leftrightarrow \xi^{t} A=0$ (just set $\eta=e_{i}$, the $i$-th unit vector in $\mathbb{K}^{n} \subseteq \mathcal{K}^{n}$ ), which in turn is equivalent with $A^{t} \xi=0$. Since $\operatorname{det}(A)=\operatorname{det}\left(A^{t}\right)$ the claim follows from (ii) $\Leftrightarrow$ (iv).

For further studies of linear algebra over the ring $\mathcal{K}$ we refer to [28]. 


\section{Generalized Metrics}

In [24], Th. 7 the following isomorphism of $\mathcal{G}(X)$-modules was established:

$$
\mathcal{G}_{s}^{r}(X) \cong L_{\mathcal{G}(X)}\left(\mathcal{G}_{1}^{0}(X)^{r}, \mathcal{G}_{0}^{1}(X)^{s} ; \mathcal{G}(X)\right) .
$$

We will make use of this identification in the following characterization result, which will motivate our definition of generalized metrics.

Theorem 3.1. Let $\hat{g} \in \mathcal{G}_{2}^{0}(X)$. The following are equivalent.

(i) For each chart $\left(V_{\alpha}, \psi_{\alpha}\right)$ and each $\tilde{x} \in\left(\psi_{\alpha}\left(V_{\alpha}\right)\right)_{c}^{\sim}\left(c f\right.$. (1.1)) the map $\hat{g}_{\alpha}(\tilde{x})$ : $\mathcal{K}^{n} \times \mathcal{K}^{n} \rightarrow \mathcal{K}^{n}$ is symmetric and nondegenerate.

(ii) $\hat{g}: \mathcal{G}_{0}^{1}(X) \times \mathcal{G}_{0}^{1}(X) \rightarrow \mathcal{G}(X)$ is symmetric and $\operatorname{det}(\hat{g})$ is invertible in $\mathcal{G}(X)$.

(iii) $\operatorname{det}(\hat{g})$ is invertible in $\mathcal{G}(X)$ and for each relatively compact open set $V \subseteq X$, there exists a representative $\left(\hat{g}_{\varepsilon}\right)_{\varepsilon}$ of $\hat{g}$ and an $\varepsilon_{0}>0$ such that $\left.\hat{g}_{\varepsilon}\right|_{V}$ is a smooth pseudo-Riemannian metric for all $\varepsilon<\varepsilon_{0}$.

Proof. (i) $\Leftrightarrow$ (ii): Supposing (i), for any $\xi, \eta \in \mathcal{G}_{0}^{1}\left(\psi_{\alpha}\left(V_{\alpha}\right)\right)$ it follows from 32, Th. 2.4 and [20, Prop. 3.8 that $\hat{g}_{\alpha}(\xi, \eta)=\hat{g}_{\alpha}(\eta, \xi)$; so $\left.\hat{g}\right|_{V_{\alpha}} \in L_{\mathcal{G}\left(V_{\alpha}\right)}\left(\mathcal{G}_{0}^{1}\left(V_{\alpha}\right), \mathcal{G}_{0}^{1}\left(V_{\alpha}\right)\right.$; $\left.\mathcal{G}\left(V_{\alpha}\right)\right)$ is symmetric. Since $L_{\mathcal{G}(-)}\left(\mathcal{G}_{1}^{0}()^{r}, \mathcal{G}_{0}^{1}\left({ }_{-}\right)^{s} ; \mathcal{G}(-)\right)$ is a sheaf, symmetry of $\hat{g}$ follows. Moreover, by 2.6 and 2.5] it follows that $\left.\operatorname{det}(\hat{g})\right|_{V_{\alpha}}$ is invertible for each $\alpha$; so $\operatorname{det}(\hat{g})$ is invertible on $X$.

The converse direction follows immediately from 2.5 and 2.6

(ii) $\Rightarrow$ (iii): We first note that for $\xi, \eta \in \mathcal{G}_{0}^{1}(X)$,

$$
\hat{g}(\xi, \eta)=\frac{1}{2}(\hat{g}(\xi, \eta)+\hat{g}(\eta, \xi))+\frac{1}{2}(\hat{g}(\xi, \eta)-\hat{g}(\eta, \xi)) .
$$

The second term in this expression is 0 in $\mathcal{G}(X)$ for all $\xi, \eta$ by assumption. Hence $\hat{g}$ equals the element of $\mathcal{G}_{2}^{0}(X)$ corresponding via (3.1) to $(\xi, \eta) \rightarrow \frac{1}{2}(\hat{g}(\xi, \eta)+\hat{g}(\eta, \xi))$. From this we obtain a representative $\left(\hat{g}_{\varepsilon}\right)_{\varepsilon}$ of $\hat{g}$ such that each $\hat{g}_{\varepsilon}: \mathfrak{X}(X) \times \mathfrak{X}(X) \rightarrow$ $\mathcal{C}^{\infty}(X)$ is symmetric. Moreover, by Proposition 2.1 for any $K \subset \subset X$ there exists $\varepsilon_{0}>0, q \in \mathbb{N}$ such that $\inf _{p \in K}\left|\operatorname{det}\left(\hat{g}_{\varepsilon}(p)\right)\right|>\varepsilon^{q}$ for $\varepsilon<\varepsilon_{0}$. In particular, each $\hat{g}_{\varepsilon}$ is nondegenerate, hence a pseudo-Riemannian metric on any open $V \subset K$.

(iii) $\Rightarrow$ (i): Let $\tilde{x} \in \psi_{\alpha}\left(V_{\alpha}\right)_{c}^{\sim}$ be supported in $K \subset \subset \psi_{\alpha}\left(V_{\alpha}\right)$ and choose a representative $\left(\hat{g}_{\varepsilon}\right)_{\varepsilon}$ of $\hat{g}$ such that each $\hat{g}_{\varepsilon}$ is a pseudo-Riemannian metric on a neighborhood of $\psi_{\alpha}^{-1}(K)$. Since each $\hat{g}_{\varepsilon}$ is symmetric, it follows that $\hat{g}_{\alpha}(\tilde{x})=\operatorname{cl}\left[\left(\hat{g}_{\alpha \varepsilon}\left(x_{\varepsilon}\right)\right)_{\varepsilon}\right]$ is symmetric as a map from $\mathcal{K}^{n} \times \mathcal{K}^{n}$ to $\mathcal{K}$. Finally, nondegeneracy follows from Lemma 2.6.

Definition 3.2. Suppose that $\hat{g} \in \mathcal{G}_{2}^{0}(X)$ satisfies one (hence all) of the equivalent conditions in Theorem 3.1 If there exists some $j \in \mathbb{N}_{0}$ such that for each relatively compact open set $V \subseteq X$ there exists a representative $\left(\hat{g}_{\varepsilon}\right)_{\varepsilon}$ of $\hat{g}$ as in 3.1 (iii) such that the index of each $\hat{g}_{\varepsilon}$ equals $j$, we call $j$ the index of $\hat{g}$.

For the above notion of index to make sense we have to establish that it does not depend on the representative of $\hat{g}$ used in (iii). To secure this property, we make use of a result from perturbation theory of finite-dimensional linear operators.

Proposition 3.3. The index of $\hat{g} \in \mathcal{G}_{2}^{0}(X)$ as introduced above is well-defined.

Proof. Let $V \subseteq X$ be relatively compact, let $\left(\hat{g}_{\varepsilon}\right)_{\varepsilon}$ be a representative of $\hat{g}$ as above and denote by $\hat{\lambda}_{\varepsilon}^{1} \geq \cdots \geq \hat{\lambda}_{\varepsilon}^{n}$ its eigenvalues. By 3.1 (iii) and 2.2 it follows that each $\hat{\lambda}^{i}$ is invertible in $\mathcal{K}$ (otherwise $\operatorname{det} \hat{g}$ would be a zero divisor). Hence each $\hat{\lambda}^{i}$ 
is strictly nonzero, i.e., there exists $r \in \mathbb{N}_{0}$ such that

$$
\left|\hat{\lambda}_{\varepsilon}^{i}\right|>\varepsilon^{r}
$$

for all $1 \leq i \leq n$ and $\varepsilon$ small. Let $\left(\tilde{g}_{\varepsilon}\right)_{\varepsilon}$ be another representative of $\hat{g}$ as in 3.1 (iii) with eigenvalues $\tilde{\lambda}_{\varepsilon}^{1} \geq \cdots \geq \tilde{\lambda}_{\varepsilon}^{n}$. By [4], 3, Th. 8.1 we have $\max _{i}\left|\tilde{\lambda}_{\varepsilon}^{i}-\hat{\lambda}_{\varepsilon}^{i}\right| \leq\left\|\tilde{g}_{\varepsilon}-\hat{g}_{\varepsilon}\right\|$. Hence from the $\mathcal{N}$-estimate we conclude that for all $i(1 \leq i \leq n),\left|\tilde{\lambda}_{\varepsilon}^{i}-\hat{\lambda}_{\varepsilon}^{i}\right|=O\left(\varepsilon^{m}\right)$ $\forall m$. Thus by (3.2) $\tilde{\lambda}_{\varepsilon}^{i}$ and $\hat{\lambda}_{\varepsilon}^{i}$ have the same sign for small $\varepsilon$.

\section{Definition 3.4.}

(i) A generalized $(0,2)$-tensor field $\hat{g} \in \mathcal{G}_{2}^{0}(X)$ is called a generalized (pseudo-) Riemannian metric if it satisfies one of the equivalent conditions in Theorem 3.1 and possesses an index (different from 0).

(ii) We call a paracompact, smooth Hausdorff manifold $X$ furnished with a generalized (pseudo-)Riemannian metric $\hat{g}$ a generalized (pseudo-)Riemannian manifold or, if the index of $\hat{g}$ is 1 or $n-1$, a generalized spacetime and denote it by $(X, \hat{g})$. The action of the metric on a pair of generalized vector fields will be denoted by $\hat{g}(\xi, \eta)$ and $\langle\xi, \eta\rangle$, equivalently.

Remark 3.5. Let us compare this notion of generalized metric with the ones introduced in the purely distributional picture in [30], 10.6, and in [34]. In [30], a distributional $(0,2)$-tensor field $g \in \mathcal{D}^{\prime}{ }_{2}(X)$ is called nondegenerate if $g(\xi, \eta)=0$ for all $\eta \in \mathfrak{X}(X)$ implies $\xi=0 \in \mathfrak{X}(X)$. However, this "nonlocal" condition is too weak to reproduce the classical notion of nondegeneracy; just take $d s^{2}=x^{2} d x^{2}$. In 34, on the other hand, $g \in \mathcal{D}^{\prime 0}(X)$ is called nondegenerate if it is nondegenerate (in the classical sense) off its singular support; so, in this approach, no statement at all is made at the singularities of the metric.

Even by combining both notions, i.e., by calling a distributional $(0,2)$-tensor field nondegenerate if it satisfies both conditions, one arrives at a comparatively weak notion. To see this, take $d s^{2}=\left(x^{2}+\delta(x)\right) d x^{2}$ on $\mathbb{R}$. This metric is easily seen to be nondegenerate in the above sense. According to 3.4, however, it depends on the "microstructure" of $\delta$, i.e., on the chosen regularization of $\delta$ whether or not $d s^{2}$ is nondegenerate in the $\mathcal{G}$-setting.

By 2.1 (i), for any representative $\left(\hat{g}_{\varepsilon}\right)_{\varepsilon}$ of a generalized pseudo-Riemannian metric, we have

$$
\forall K \subset \subset X \exists m \in \mathbb{N} \exists \varepsilon_{0}>0: \inf _{p \in K}\left|\operatorname{det} \hat{g}_{\varepsilon}(p)\right| \geq \varepsilon^{m} \forall \varepsilon<\varepsilon_{0} .
$$

Proposition 3.6. Let $(X, \hat{g})$ be a generalized pseudo-Riemannian manifold and let $\hat{g}=\operatorname{cl}\left[\left(\hat{g}_{\varepsilon}\right)_{\varepsilon}\right]$. Then the inverse metric $\hat{g}^{-1}:=\operatorname{cl}\left[\left(\hat{g}_{\varepsilon}^{-1}\right)_{\varepsilon}\right]$ is a well-defined element of $\mathcal{G}_{0}^{2}(X)$.

Proof. We first note that by (3.3), in any relatively compact chart $V$ and for fixed $\varepsilon<\varepsilon_{0}(V)$ we may define (in the usual notation) $\hat{g}^{i j}{ }_{\varepsilon}$ to be the pointwise inverse of $\hat{g}_{i j \varepsilon}$ which is obviously a smooth $(2,0)$-tensor field. By the cofactor formula of matrix inversion we have $\hat{g}^{i j}{ }_{\varepsilon}=\operatorname{cof}\left(\hat{g}_{i j \varepsilon}\right) /\left(\operatorname{det} \hat{g}_{i j \varepsilon}\right)$; so from (3.3) we conclude that $\left(\hat{g}^{j i}{ }_{\varepsilon}\right)_{\varepsilon}$ is moderate on its domain of definition. By the same reasoning, choosing a different representative of $\hat{g}$ in this calculation perturbs $\hat{g}^{i j}$ merely by an element of $\mathcal{N}$. From the sheaf property of $\mathcal{G}_{0}^{2}(-)$ the result follows. 
From now on we denote the inverse metric (using abstract index notation, cf. Section (1) by $\hat{g}^{a b}$, its components by $\hat{g}^{i j}$ and the components of a representative by $\hat{g}_{\varepsilon}^{i j}$. Also, we shall denote the line element by $\hat{d s}^{2}=\operatorname{cl}\left[\left(\hat{d}_{\varepsilon}^{2}\right)_{\varepsilon}\right]$.

\section{Example 3.7.}

(i) A sufficient condition for a sequence $\left(g_{\varepsilon}\right)_{\varepsilon}$ of classical (smooth) metrics of constant index to constitute a representative of a generalized metric - apart from being moderate - is to be zero-associated (i.e., to converge locally uniformly) to a classical (then necessarily continuous) metric $g$. Indeed, (3.3) is satisfied in this case since $\operatorname{det} g_{\varepsilon} \rightarrow \operatorname{det} g$ uniformly on compact sets; so the claim follows from 3.1 (iii).

(ii) The metric of a two-dimensional cone was modelled in 6 by a generalized metric (in the full setting) obtained through embedding via convolution.

(iii) The line element of impulsive pp-waves in [39], [22, [23] was modelled by

$$
\hat{d s}^{2}=f(x, y) D(u) d u^{2}-d u d v+d x^{2}+d y^{2},
$$

where $D$ denotes a generalized delta function which allows for a strict delta net as a representative (see Section [6) and $f$ is a smooth function.

(iv) Further examples may be found e.g., in [2], [38], [29].

Since taking the determinant is a polynomial operation, we cannot expect association to be compatible with inverting a metric. However, the analogous statement for $k$-association holds by an application of [24], Prop. 3 (ii).

Proposition 3.8. Let $\hat{g}_{a b}$ be a generalized metric and $\hat{g}_{a b} \approx_{k} g_{a b}$, where $g_{a b}$ is a classical $\mathcal{C}^{k}$-pseudo-Riemannian metric. Then $\hat{g}^{a b} \approx_{k} g^{a b}$.

Additional important properties of generalized metrics are presented in the following result.

Proposition 3.9. Let $(X, \hat{g})$ be a generalized pseudo-Riemannian manifold.

(i) $\xi \in \mathcal{G}_{0}^{1}(X), \hat{g}(\xi, \eta)=0 \forall \eta \in \mathcal{G}_{0}^{1}(X) \Rightarrow \xi=0$.

(ii) $\hat{g}$ induces a $\mathcal{G}(X)$-linear isomorphism $\mathcal{G}_{0}^{1}(X) \rightarrow \mathcal{G}_{1}^{0}(X)$ by $\xi \mapsto \hat{g}(\xi$, . ).

Proof. (i) We have to show that for $\hat{g}=\operatorname{cl}\left[\left(\hat{g}_{\varepsilon}\right)_{\varepsilon}\right], \xi=\operatorname{cl}\left[\left(\xi_{\varepsilon}\right)_{\varepsilon}\right],\left(\hat{g}_{\varepsilon}\left(\xi_{\varepsilon}, \eta_{\varepsilon}\right)\right)_{\varepsilon} \in \mathcal{N}(X)$ for all $\eta=\operatorname{cl}\left[\left(\eta_{\varepsilon}\right)_{\varepsilon}\right]$ implies $\left(\xi_{\varepsilon}\right)_{\varepsilon} \in(\mathcal{N})_{0}^{1}(X)$. By the sheaf properties of $\mathcal{G}_{s}^{r}(-)$, it suffices to establish the claim locally on every chart $V_{\alpha}$, i.e.,

$$
\left(\hat{g}_{i j \varepsilon} \xi_{\varepsilon}^{i} \eta_{\varepsilon}^{j}\right)_{\varepsilon} \in \mathcal{N}\left(V_{\alpha}\right) \forall\left(\eta_{\varepsilon}^{j}\right)_{\varepsilon} \Rightarrow\left(\xi_{\varepsilon}^{i}\right)_{\varepsilon} \in \mathcal{N}\left(V_{\alpha}\right) \quad i=1, \ldots, n .
$$

Setting $\eta_{\varepsilon}^{j}=\sum_{l} \hat{g}^{j l}{ }_{\varepsilon} \xi_{\varepsilon}^{l}$ gives $\left(\sum_{i j} \hat{g}_{i j \varepsilon} \xi_{\varepsilon}^{i} \eta_{\varepsilon}^{j}\right)=\sum_{i}\left(\xi_{\varepsilon}^{i}\right)^{2}$. It follows that each $\xi^{i}$ satisfies the $\mathcal{N}$-estimates of order 0 . Thus the claim follows from [12], Th. 13.1.

(ii) By (3.1) $\xi^{*}:=\hat{g}(\xi,$.$) is indeed a one-form and the assignment \xi \mapsto \xi^{*}$ is $\mathcal{G}(X)$ linear. Moreover, injectivity of this map follows from 3.9 (i). It remains to show that the assignment is onto. Locally, any generalized one-form can be written as $A=A_{i} d x^{i}$. Define a generalized vector field by $V=\hat{g}^{j k} A_{j} \partial_{k}$. Then

$$
\left\langle V, \partial_{l}\right\rangle=\hat{g}^{j k} A_{k}\left\langle\partial_{j}, \partial_{l}\right\rangle=A_{l}
$$

and the result again follows from the sheaf property of $\mathcal{G}_{0}^{1}(-)$.

The isomorphism in (ii) above - as in the classical context - extends naturally to generalized tensor fields of higher types. Hence from now on, we shall use the common conventions on upper and lower indices also in the context of generalized 
tensor fields. In particular, identifying a vector field $\xi^{a} \in \mathcal{G}_{0}^{1}(X)$ with its metrically equivalent one-form $\xi_{a}$, we denote its contravariant respectively covariant components by $\xi^{i}$ and $\xi_{i}$. A similar convention will apply to representatives.

\section{Sections on a Generalized Mapping}

In 20, the space $\mathcal{G}[X, Y]$ of Colombeau generalized functions on the manifold $X$ taking values in the manifold $Y$ as well as the space $\operatorname{Hom}_{\mathcal{G}}[E, F]$ of generalized vector bundle homomorphisms from $E$ to $F$ was defined. In order to obtain a consistent description of geodesics of generalized pseudo-Riemannian metrics, we need some additional constructions extending the framework introduced there. The present section is devoted to the development of these concepts, and we shall freely use notations and definitions from [20]. For the convenience of the reader, however, we recall the definition of generalized functions valued in a manifold.

Definition 4.1. Let $X, Y$ be paracompact, smooth Hausdorff manifolds.

(a) The space $\mathcal{E}_{M}[X, Y]$ of compactly bounded (c-bounded, for short) moderate maps from $X$ to $Y$ is defined as the set of all $\left(u_{\varepsilon}\right)_{\varepsilon} \in \mathcal{C}^{\infty}(X, Y)^{(0,1]}$ such that

(i) $\forall K \subset \subset \Omega \exists \varepsilon_{0}>0 \exists K^{\prime} \subset \subset Y \forall \varepsilon<\varepsilon_{0}: u_{\varepsilon}(K) \subseteq K^{\prime}$.

(ii) $\forall k \in \mathbb{N}$, for each chart $(V, \varphi)$ in $X$, each chart $(W, \psi)$ in $Y$, each $L \subset \subset V$ and each $L^{\prime} \subset \subset W$ there exists $N \in \mathbb{N}$ with

$$
\sup _{p \in L \cap u_{\varepsilon}^{-1}\left(L^{\prime}\right)}\left\|D^{(k)}\left(\psi \circ u_{\varepsilon} \circ \varphi^{-1}\right)(\varphi(p))\right\|=O\left(\varepsilon^{-N}\right) .
$$

(b) Two elements $\left(u_{\varepsilon}\right)_{\varepsilon},\left(v_{\varepsilon}\right)_{\varepsilon}$ of $\mathcal{E}_{M}[X, Y]$ are called equivalent, $\left(u_{\varepsilon}\right)_{\varepsilon} \sim\left(v_{\varepsilon}\right)_{\varepsilon}$, if the following conditions are satisfied:

(i) for all $K \subset \subset X, \sup _{p \in K} d_{h}\left(u_{\varepsilon}(p), v_{\varepsilon}(p)\right) \rightarrow 0(\varepsilon \rightarrow 0)$ for some (hence every) Riemannian metric $h$ on $Y$;

(ii) $\forall k \in \mathbb{N}_{0} \forall m \in \mathbb{N}$, for each chart $(V, \varphi)$ in $X$, each chart $(W, \psi)$ in $Y$, each $L \subset \subset V$ and each $L^{\prime} \subset \subset W$ :

$$
\sup _{-1}\left\|D^{(k)}\left(\psi \circ u_{\varepsilon} \circ \varphi^{-1}-\psi \circ v_{\varepsilon} \circ \varphi^{-1}\right)(\varphi(p))\right\|=O\left(\varepsilon^{m}\right) .
$$

(c) The quotient $\mathcal{G}[X, Y]:=\mathcal{E}_{M}[X, Y] / \sim$ is called the space of Colombeau generalized functions on $X$ valued in $Y$.

The space $\operatorname{Hom}_{\mathcal{G}}[E, F]$ of generalized vector bundle homomorphisms is defined along the same lines (see [20], Section 3). Here we introduce a "hybrid" variant of generalized mappings defined on a manifold and taking values in a vector bundle.

Definition 4.2. Let $\left(F, \pi_{Y}, Y\right)$ be a vector bundle and denote by $\mathcal{E}_{M}^{h}[X, F]$ the set of all nets $\left(u_{\varepsilon}\right)_{\varepsilon} \in \mathcal{C}^{\infty}(X, F)^{(0,1]}$ satisfying (with $\underline{u_{\varepsilon}}:=\pi_{Y} \circ u_{\varepsilon}$ )

(i) $\forall K \subset \subset X \exists K^{\prime} \subset \subset Y \exists \varepsilon_{0}>0 \forall \varepsilon<\varepsilon_{0} u_{\varepsilon}(\bar{K}) \subseteq K^{\prime}$;

(ii) $\forall k \in \mathbb{N}_{0} \forall(V, \varphi)$ chart in $X \forall(W, \Psi)$ vector bundle chart in $F \forall L \subset \subset$ $V \forall L^{\prime} \subset \subset W \exists N \in \mathbb{N} \exists \varepsilon_{1}>0 \exists C>0$ such that

$$
\left\|D^{(k)}\left(\Psi \circ u_{\varepsilon} \circ \varphi^{-1}\right)(\varphi(p))\right\| \leq C \varepsilon^{-N}
$$

for each $\varepsilon<\varepsilon_{1}$ and each $p \in L \cap{\underline{u_{\varepsilon}}}^{-1}\left(L^{\prime}\right)$.

In particular, $\left(u_{\varepsilon}\right)_{\varepsilon} \in \mathcal{E}_{M}^{h}[X, F]$ implies $\left(\underline{u_{\varepsilon}}\right)_{\varepsilon} \in \mathcal{E}_{M}[X, Y]$.

Definition 4.3. $\left(u_{\varepsilon}\right)_{\varepsilon},\left(v_{\varepsilon}\right)_{\varepsilon} \in \mathcal{E}_{M}^{h}[X, F]$ are called equivalent, $\left(u_{\varepsilon}\right)_{\varepsilon} \sim_{h}\left(v_{\varepsilon}\right)_{\varepsilon}$, if the following conditions are satisfied: 
(i) for each $K \subset \subset X, \sup _{p \in X} d_{h}\left(\underline{u_{\varepsilon}}, \underline{v_{\varepsilon}}\right) \rightarrow 0$ for some (hence every) Riemannian metric $h$ on $Y$;

(ii) $\forall k \in \mathbb{N}_{0} \forall m \in \mathbb{N} \forall(V, \varphi)$ chart in $X, \forall(W, \Psi)$ vector bundle chart in $F$, $\forall L \subset \subset V \forall L^{\prime} \subset \subset W \exists \varepsilon_{1}>0 \exists C>0$ such that

$$
\left\|D^{(k)}\left(\Psi \circ u_{\varepsilon} \circ \varphi^{-1}-\Psi \circ v_{\varepsilon} \circ \varphi^{-1}\right)(\varphi(p))\right\| \leq C \varepsilon^{m}
$$

for each $\varepsilon<\varepsilon_{1}$ and each $p \in L \cap \underline{u}^{-1}\left(L^{\prime}\right) \cap \underline{v}_{\varepsilon}^{-1}\left(L^{\prime}\right)$.

Since $\operatorname{pr}_{1} \circ \Psi \circ u_{\varepsilon} \circ \varphi^{-1}=\psi \circ \underline{u_{\varepsilon}} \circ \varphi^{-1}\left(\right.$ with $\left.\operatorname{pr}_{1}: \psi(W) \times \mathbb{R}^{n^{\prime}} \rightarrow \psi(W)\right)$, it follows that 4.3 (i) precisely means that $\left(u_{\varepsilon}\right)_{\varepsilon} \sim\left(v_{\varepsilon}\right)_{\varepsilon}$ in $\mathcal{E}_{M}[X, Y]$. Moreover, by the same methods as employed in [20, Remarks 2.4 and 2.6 we conclude that both moderateness and equivalence can be formulated equivalently by merely requiring 4.2 resp. 4.3 for charts from any given atlas of $X$ resp. vector bundle atlas of $F$.

Definition 4.4. The space of hybrid Colombeau generalized functions from the manifold $X$ into the vector bundle $F$ is defined by

$$
\mathcal{G}^{h}[X, F]:=\mathcal{E}_{M}^{h}[X, F] / \sim_{h} .
$$

\section{Theorem 4.5.}

(i) Let $u=\operatorname{cl}\left[\left(u_{\varepsilon}\right)_{\varepsilon}\right] \in \mathcal{G}[X, Y], v=\operatorname{cl}\left[\left(v_{\varepsilon}\right)_{\varepsilon}\right] \in \Gamma_{\mathcal{G}}(Y, F)$. Then $v \circ u:=\operatorname{cl}\left[\left(v_{\varepsilon} \circ\right.\right.$ $\left.\left.u_{\varepsilon}\right)_{\varepsilon}\right]$ is a well-defined, element of $\mathcal{G}^{h}[X, F]$.

(ii) Let $u=\operatorname{cl}\left[\left(u_{\varepsilon}\right)_{\varepsilon}\right] \in \Gamma_{\mathcal{G}}(X, E), v=\operatorname{cl}\left[\left(v_{\varepsilon}\right)_{\varepsilon}\right] \in \operatorname{Hom}_{\mathcal{G}}[E, F]$. Then $v \circ u:=$ $\operatorname{cl}\left[\left(v_{\varepsilon} \circ u_{\varepsilon}\right)_{\varepsilon}\right]$ is a well-defined element of $\mathcal{G}^{h}[X, F]$.

Proof. (i) We first have to show that $\left(v_{\varepsilon} \circ u_{\varepsilon}\right)_{\varepsilon} \in \mathcal{E}_{M}^{h}[X, F]$. Property (i) of 4.2 is obvious. Let $(V, \varphi)$ be a chart in $X, L \subset \subset V,(W, \Psi)$ a vector bundle chart in $F, L^{\prime} \subset \subset W$ and $p \in L$ such that $v_{\varepsilon} \circ u_{\varepsilon}(p)=u_{\varepsilon}(p) \in L^{\prime}$. Then $D^{(k)}\left(\Psi \circ v_{\varepsilon} \circ\right.$ $\left.u_{\varepsilon} \circ \varphi^{-1}\right)(\varphi(p))=D^{(k)}\left(\left(\Psi \circ v_{\varepsilon} \circ \psi^{-1}\right) \circ\left(\psi \circ u_{\varepsilon} \circ \varphi^{-1}\right)\right)(\varphi(p))$ can immediately be estimated using moderateness of $\left(u_{\varepsilon}\right)_{\varepsilon}$ and $\left(v_{\varepsilon}\right)_{\varepsilon}$.

To show that $v \circ u$ is well-defined, let $\left(u_{\varepsilon}\right)_{\varepsilon} \sim\left(u_{\varepsilon}^{\prime}\right)_{\varepsilon}$ Then $\left(\underline{v_{\varepsilon} \circ u_{\varepsilon}}\right)_{\varepsilon}=\left(u_{\varepsilon}\right)_{\varepsilon} \sim$ $\left(u_{\varepsilon}^{\prime}\right)_{\varepsilon}=\left(v_{\varepsilon} \circ u_{\varepsilon}^{\prime}\right)_{\varepsilon}$, which verifies (i) of 4.3 To show (ii), let $L^{\prime}$ be such that $u_{\varepsilon}(L) \cup u_{\varepsilon}^{\prime(L) \subseteq} L^{\prime}$ for $\varepsilon$ small, cover $L^{\prime}$ by charts $\left(W_{j}, \psi_{j}\right)(1 \leq j \leq r)$ and write $L^{\prime}=\bigcup_{j=1}^{r} L_{j}^{\prime}$ with $L_{j}^{\prime} \subset \subset \overline{W_{j}^{\prime}} \subset \subset W_{j}$ where $W_{j}^{\prime}$ is an open neighborhood of $L_{j}^{\prime}$. Then for $\varepsilon$ small and $p \in L$ with $u_{\varepsilon}(p), u_{\varepsilon}^{\prime}(p) \in L^{\prime}$ there exists $j$ with $u_{\varepsilon}(p), u_{\varepsilon}^{\prime}(p) \in W_{j}^{\prime}$. Hence

$$
\begin{aligned}
D^{(k)}\left(\Psi \circ v_{\varepsilon} \circ u_{\varepsilon} \circ \varphi^{-1}-\Psi \circ v_{\varepsilon} \circ u_{\varepsilon}^{\prime} \circ \varphi^{-1}\right)(\varphi(p)) \\
=D^{(k)}\left(\left(\Psi \circ \Psi_{j}^{-1}\right) \circ\left(\Psi_{j} \circ v_{\varepsilon} \circ \psi_{j}^{-1}\right) \circ\left(\psi_{j} \circ u_{\varepsilon} \circ \varphi^{-1}\right)\right. \\
\left.\quad-\left(\Psi \circ \Psi_{j}^{-1}\right) \circ\left(\Psi_{j} \circ v_{\varepsilon} \circ \psi_{j}^{-1}\right) \circ\left(\psi_{j} \circ u_{\varepsilon}^{\prime} \circ \varphi^{-1}\right)\right)(\varphi(p)) .
\end{aligned}
$$

Since the norm of any derivative of each $\Psi_{j} \circ v_{\varepsilon} \circ \psi_{j}^{-1}$ is bounded by some inverse power of $\varepsilon$ uniformly on $W_{j}^{\prime}(1 \leq j \leq r),\left(v_{\varepsilon} \circ u_{\varepsilon}\right) \sim_{h}\left(v_{\varepsilon} \circ u_{\varepsilon}^{\prime}\right)_{\varepsilon}$ follows from the above equality and $\left(u_{\varepsilon}\right)_{\varepsilon} \sim\left(u_{\varepsilon}^{\prime}\right)_{\varepsilon}$ using [20], Lemma 2.5 .

Finally, let $\left(v_{\varepsilon}-v_{\varepsilon}^{\prime}\right)_{\varepsilon} \in \Gamma_{\mathcal{N}}(Y, F)$. We have to show that $\left(v_{\varepsilon} \circ u_{\varepsilon}\right)_{\varepsilon} \sim_{h}\left(v_{\varepsilon}^{\prime} \circ u_{\varepsilon}\right)_{\varepsilon}$. In this case, (i) of 4.3 is satisfied trivially. Concerning (ii), let $L \subset \subset V, L^{\prime} \subset \subset W$, $p \in L \cap u_{\varepsilon}^{-1}\left(L^{\prime}\right)$. Then $\left\|D^{(k)}\left(\Psi \circ\left(v_{\varepsilon}-v_{\varepsilon}^{\prime}\right) \circ \psi^{-1}\right) \circ\left(\psi \circ u_{\varepsilon} \circ \varphi^{-1}\right)\right\|$ can be estimated using the $\mathcal{N}$-bounds for $\left(v_{\varepsilon}-v_{\varepsilon}^{\prime}\right)_{\varepsilon}$ on $L^{\prime}$ and moderateness of $\left(u_{\varepsilon}\right)_{\varepsilon}$, yielding the claim.

(ii) In this part of the proof we only record the general structure of the terms to be estimated and do not embark on the topological arguments (which, anyways, 
are of a simpler nature than in (i)). Since in this case $\underline{v_{\varepsilon} \circ u_{\varepsilon}}=\underline{v_{\varepsilon}}$, (i) of 4.2 is again obvious. Moderateness now follows by estimating terms of the form $D^{(k)}\left(\left(\Psi \circ v_{\varepsilon} \circ\right.\right.$ $\left.\left.\Phi^{-1}\right) \circ\left(\Phi \circ u_{\varepsilon} \circ \varphi^{-1}\right)\right)$. To see that the composition is well-defined, suppose first that $\left(u_{\varepsilon}\right)_{\varepsilon}-\left(u_{\varepsilon}^{\prime}\right)_{\varepsilon} \in \Gamma_{\mathcal{N}}(X, E)$. Now we note that (writing $\Phi \circ u_{\varepsilon} \circ \varphi^{-1}=(x \mapsto$ $\left.\left.\left(x, u_{\varepsilon \Phi}^{(2)}(x)\right)\right)\right)$

$$
\left(\Psi \circ v_{\varepsilon} \circ \Phi^{-1}\right) \circ\left(\Phi \circ u_{\varepsilon} \circ \varphi^{-1}\right)(x)=\left(v_{\varepsilon \Psi \Phi}^{(1)}(x), v_{\varepsilon \Psi \Phi}^{(2)}(x) u_{\varepsilon \Phi}^{(2)}(x)\right) .
$$

Hence we have to estimate $v_{\varepsilon \Psi \Phi}^{(2)}(x)\left(u_{\varepsilon \Phi}^{(2)}(x)-u_{\varepsilon \Phi}^{\prime(2)}(x)\right)$, which is immediate from our assumption on $\left(u_{\varepsilon}\right)_{\varepsilon},\left(u_{\varepsilon}^{\prime}\right)_{\varepsilon}$. Finally, let $\left(v_{\varepsilon}\right)_{\varepsilon} \sim_{v b}\left(v_{\varepsilon}^{\prime}\right)_{\varepsilon}$. Then to show equivalence of $\left(v_{\varepsilon} \circ u_{\varepsilon}\right)_{\varepsilon}$ and $\left(v_{\varepsilon}^{\prime} \circ u_{\varepsilon}\right)_{\varepsilon}$, by (4.1) it suffices to consider $\left(v_{\varepsilon \Psi \Phi}^{(2)}(x)-v_{\varepsilon \Psi \Phi}^{\prime(2)}(x)\right) u_{\varepsilon \Phi}^{(2)}(x)$; so the claim follows.

Using $\mathcal{G}^{h}$ we now introduce the notion of generalized sections along generalized maps, and, in particular, of generalized vector fields on generalized maps.

Definition 4.6. For each $u \in \mathcal{G}[X, Y]$, we will denote by $\left(\mathcal{G}^{h}[X, F]\right)(u)$ the set $\left\{\xi \in \mathcal{G}^{h}[X, F] \mid \underline{\xi}=u\right\}$. In particular, a generalized vector field $\xi$ on $u \in \mathcal{G}[X, Y]$ is an element of

$$
\mathfrak{X}_{\mathcal{G}}(u):=\left\{\xi \in \mathcal{G}^{h}[X, T Y] \mid \underline{\xi}=u\right\}
$$

\section{Generalized Pseudo-Riemannian Geometry}

The aim of this section is to initiate a study of pseudo-Riemannian geometry in the present setting. We start by introducing the notion of a generalized connection and its Christoffel symbols.

\section{Definition 5.1.}

(i) A generalized connection $\hat{D}$ on a manifold $X$ is a map $\mathcal{G}_{0}^{1}(X) \times \mathcal{G}_{0}^{1}(X) \rightarrow \mathcal{G}_{0}^{1}(X)$ satisfying

(D1) $\hat{D}_{\xi} \eta$ is $\mathcal{R}$-linear in $\eta$;

(D2) $\hat{D}_{\xi} \eta$ is $\mathcal{G}(X)$-linear in $\xi$;

(D3) $\hat{D}_{\xi}(u \eta)=u \hat{D}_{\xi} \eta+\xi(u) \eta$ for all $u \in \mathcal{G}(X)$.

(ii) Let $\left(V_{\alpha}, \psi_{\alpha}\right)$ be a chart on $X$ with coordinates $x^{i}$. We define the generalized Christoffel symbols for this chart to be the $n^{3}$ functions $\hat{\Gamma}_{i j}^{k} \in \mathcal{G}\left(V_{\alpha}\right)$ given by

$$
\hat{D}_{\partial_{i}} \partial_{j}=\sum_{k} \hat{\Gamma}_{i j}^{k} \partial_{k} \quad 1 \leq i, j \leq n .
$$

Since $\mathcal{C}^{\infty}(X)$ is a submodule of $\mathcal{G}(X)$ and the sheaf $\mathcal{G}(X)$ is fine, (D2) and (D3) in particular imply localizability of any generalized connection with respect to its arguments.

We are now in the position to prove the "Fundamental Lemma of (pseudo)Riemannian Geometry" in the present setting.

Theorem 5.2. Let $(X, \hat{g})$ be a generalized pseudo-Riemannian manifold. Then there exists a unique generalized connection $\hat{D}$ such that

(D4) $[\xi, \eta]=\hat{D}_{\xi} \eta-\hat{D}_{\eta} \xi$ and

(D5) $\xi \hat{g}(\eta, \zeta)=\hat{g}\left(\hat{D}_{\xi} \eta, \zeta\right)+\hat{g}\left(\eta, \hat{D}_{\xi} \zeta\right)$ 
hold for all $\xi, \eta, \zeta$ in $\mathcal{G}_{0}^{1}(X) . \hat{D}$ is called the generalized Levi-Civita connection of $X$ and is characterized by the Koszul formula

$$
\begin{aligned}
2 \hat{g}\left(\hat{D}_{\xi} \eta, \zeta\right)= & \xi \hat{g}(\eta, \zeta)+\eta \hat{g}(\zeta, \xi)-\zeta \hat{g}(\xi, \eta) \\
& -\hat{g}(\xi,[\eta, \zeta])+\hat{g}(\eta,[\zeta, \xi])+\hat{g}(\zeta,[\xi, \eta]) .
\end{aligned}
$$

Proof. Assume $\hat{D}$ to be a generalized connection additionally satisfying (D4) and (D5). As in the classical proof (see, e.g., 33, $\S 3$, Theorem 11) using the latter two properties one shows that equation (5.1) is satisfied and by the injectivity of the map in 3.9 (ii), uniqueness follows.

To show existence, define $F(\xi, \eta, \zeta)$ to be one half the right-hand side of (5.1). Then for fixed $\eta, \zeta$ the function $\xi \mapsto F(\xi, \eta, \zeta)$ is $\mathcal{G}(X)$-linear, hence defines a generalized one-form (using (3.1)). Again by [3.9] (ii) there exists a unique generalized vector field metrically equivalent to this one-form which we may call $\hat{D}_{\xi} \eta$. Now it is easy to derive (D1)-(D5) along the lines of the classical proof just using the bilinearity of $g$ and the standard properties of the Lie bracket (cf. [24], the remark following Def. 10).

As in the classical case from the torsion-free condition (i.e., (D4)) we immediately infer the symmetry of the Christoffel symbols of the Levi-Civita connection in the lower pair of indices. Moreover, from (D3) and the Koszul formula (5.1) we derive (analogously to the classical case) the following.

Proposition 5.3. Given a chart as in 5.1 (ii) we have for the generalized LeviCivita connection $\hat{D}$ of $(X, \hat{g})$ and any vector field $\xi \in \mathcal{G}_{0}^{1}(X)$,

$$
\hat{D}_{\partial_{i}}\left(\xi^{j} \partial_{j}\right)=\left(\frac{\partial \xi^{k}}{\partial x^{i}}+\hat{\Gamma}_{i j}^{k} \xi^{j}\right) \partial_{k} .
$$

Moreover, the generalized Christoffel symbols are given by

$$
\hat{\Gamma}_{i j}^{k}=\frac{1}{2} \hat{g}^{k m}\left(\frac{\partial \hat{g}_{j m}}{\partial x^{i}}+\frac{\partial \hat{g}_{i m}}{\partial x^{j}}-\frac{\partial \hat{g}_{i j}}{\partial x^{m}}\right) .
$$

In particular, we see that we could equivalently have introduced the generalized Christoffel symbols of a generalized metric by demanding the classical formula on the level of representatives.

To state the next result concerning consistency properties of generalized connections resp. generalized Christoffel symbols with respect to their classical counterparts, we need to define the action of a classical (smooth) connection $D$ on generalized vector fields $\xi, \eta$. This is done by setting

$$
D_{\xi} \eta:=\operatorname{cl}\left[\left(D_{\xi_{\varepsilon}} \eta_{\varepsilon}\right)_{\varepsilon}\right]
$$

which is easily seen to be independent of the representatives chosen for $\xi$ and $\eta$. From the local formulae in the above proposition and [24], Prop. 3, we conclude

Proposition 5.4. Let $(X, \hat{g})$ be a generalized pseudo-Riemannian manifold.

(i) If $\hat{g}_{a b}=\Sigma\left(g_{a b}\right)$, where $g_{a b}$ is a classical smooth pseudo-Riemannian metric, then we have, in any chart, $\hat{\Gamma}_{j k}^{i}=\sigma\left(\Gamma_{j k}^{i}\right)$ (with $\Gamma_{j k}^{i}$ denoting the Christoffel Symbols of $g$ ). Hence for all $\xi, \eta \in \mathcal{G}_{0}^{1}(X)$,

$$
\hat{D}_{\xi} \eta=D_{\xi} \eta \text {. }
$$


(ii) If $\hat{g}_{a b} \approx_{\infty} g_{a b}, g_{a b}$ a classical smooth metric, $\xi, \eta \in \mathcal{G}_{0}^{1}(X)$ and $\xi \approx_{\infty} \zeta \in$ $\mathcal{T}_{0}^{1}(X), \eta \approx \nu \in \mathcal{D}_{0}^{\prime 1}(X)$ (or vice versa, i.e., $\xi \approx \zeta \in \mathcal{D}_{0}^{\prime 1}(X), \eta \approx_{\infty} \nu \in$ $\left.\mathcal{T}_{0}^{1}(X)\right)$, then

$$
\hat{D}_{\xi} \eta \approx D_{\zeta} \nu
$$

(iii) Let $\hat{g}_{a b} \approx_{k} g_{a b}, g_{a b}$ a classical $\mathcal{C}^{k}$-metric. Then, in any chart, $\hat{\Gamma}_{j k}^{i} \approx_{k-1}$ $\Gamma_{j k}^{i}$. If in addition $\xi, \eta \in \mathcal{G}_{0}^{1}(X), \xi \approx_{k-1} \zeta \in \Gamma^{k-1}(X, T X)$ and $\eta \approx_{k} \nu \in$ $\Gamma^{k}(X, T X)$, then

$$
\hat{D}_{\xi} \eta \approx_{k-1} D_{\zeta} \nu
$$

Our next aim is to define the induced covariant derivative of a generalized metric on a generalized curve. Let $J \subseteq \mathbb{R}$ be an interval and $\gamma \in \mathcal{G}[J, X]$. Let $\hat{g} \in \mathcal{G}_{2}^{0}(X)$ be a generalized metric. For any $K \subset \subset J$ there exists $\varepsilon_{0}>0$ and $K^{\prime} \subset \subset X$ such that $\gamma_{\varepsilon}(K) \subseteq K^{\prime}$ for $\varepsilon<\varepsilon_{0}$. According to 3.1 we may choose a representative $\left(\hat{g}_{\varepsilon}\right)_{\varepsilon}$ of $\hat{g}$ such that each $\hat{g}_{\varepsilon}$ is a pseudo-Riemannian metric in a neighborhood of $K^{\prime}$. Let $\xi=\operatorname{cl}\left[\left(\xi_{\varepsilon}\right)_{\varepsilon}\right] \in \mathfrak{X}_{\mathcal{G}}(\gamma)$. For each fixed small $\varepsilon$, we let $\xi_{\varepsilon}^{\prime}$ be the induced covariant derivative of $\xi_{\varepsilon}$ on $\gamma_{\varepsilon}$ with respect to $\hat{g}_{\varepsilon}$.

Definition 5.5. We call $\xi^{\prime}:=\operatorname{cl}\left[\left(\xi_{\varepsilon}^{\prime}\right)_{\varepsilon}\right] \in \mathfrak{X}_{\mathcal{G}}(\gamma)$ the induced covariant derivative of $\xi$ on $\gamma$ with respect to $\hat{g}$.

For this definition to make sense, we have to show that $\xi^{\prime}$ is independent of the chosen representatives $\left(\gamma_{\varepsilon}\right)_{\varepsilon},\left(\xi_{\varepsilon}\right)_{\varepsilon}$ and $\left(\hat{g}_{\varepsilon}\right)_{\varepsilon}$. To this end we note that for fixed $\varepsilon$, the local form of $\xi_{\varepsilon}^{\prime}$ is given by (with $\hat{\Gamma}_{\varepsilon i j}^{k}$ the Christoffel symbols of $\hat{g}_{\varepsilon}$ )

$$
\xi_{\varepsilon}^{\prime}=\sum_{k}\left(\frac{d \xi_{\varepsilon}^{k}}{d t}+\sum_{i, j} \hat{\Gamma}_{\varepsilon i j}^{k} \frac{d \gamma_{\varepsilon}^{i}}{d t} \xi_{\varepsilon}^{j}\right) \partial_{k} .
$$

From this, on the one hand, we conclude that $\left(\xi_{\varepsilon}^{\prime}\right)_{\varepsilon}$ is indeed moderate and, on the other hand, it is straightforward to check that choosing different representatives for $\xi, \gamma$ or $\hat{g}$ does not change the class of $\xi^{\prime}$ in $\mathfrak{X}_{\mathcal{G}}(\gamma)$. Finally, we conclude that the above restriction of $\gamma$ to relatively compact subintervals of $J$ can be overcome by "patching together" the representatives of $\xi^{\prime}$ obtained for a covering of $J$ by relatively compact subintervals. In fact, these partially defined generalized functions coincide on overlapping intervals again due to the explicit local form (5.2).

The main properties of the induced covariant derivative are collected in the following result.

Proposition 5.6. Let $\hat{g}$ be a generalized metric on $X$ with Levi-Civita connection $\hat{D}$ and let $\gamma \in \mathcal{G}[J, X]$. Then

(i) $\left(\tilde{r} \xi_{1}+\tilde{s} \xi_{2}\right)^{\prime}=\tilde{r} \xi_{1}^{\prime}+\tilde{s} \xi_{2}^{\prime} \quad\left(\tilde{r}, \tilde{s} \in \mathcal{K}, \xi_{1}, \xi_{2} \in \mathfrak{X}_{\mathcal{G}}(\gamma)\right)$;

(ii) $(u \xi)^{\prime}=\frac{d u}{d t} \xi+u \xi^{\prime} \quad\left(u \in \mathcal{G}(J), \xi \in \mathfrak{X}_{\mathcal{G}}(\gamma)\right)$;

(iii) $(\xi \circ \gamma)^{\prime}=\hat{D}_{\gamma^{\prime}(.)} \xi \quad$ in $\mathfrak{X}_{\mathcal{G}}(\gamma)\left(\xi \in \mathcal{G}_{0}^{1}(X)\right)$.

Proof. The composition $\xi \circ \gamma$ in (iii) is well-defined by 4.5 (i). Moreover, the righthand side of (iii) exists since $\xi \rightarrow \hat{D}_{\xi} \eta$ is a (vector-valued) generalized tensor field (cf. (D2) in 5.1). All the claimed identities now follow directly from the local form (5.2) of the induced covariant derivative.

On $J$ we consider the section $s:=\left.t \mapsto \frac{d}{d t}\right|_{t} \equiv(t, 1)$. Applying [20], Def. 3.3 to a generalized curve $\gamma \in \mathcal{G}[J, X]$, it follows from 4.5 (ii) that its velocity vector field 
defined by $\gamma^{\prime}:=T \gamma \circ s$ is a well-defined element of $\mathfrak{X}_{\mathcal{G}}(\gamma)$. Then $\gamma^{\prime \prime}$ is defined as the induced covariant derivative of $\gamma^{\prime}$ on $\gamma$.

Definition 5.7. A geodesic in a generalized pseudo-Riemannian manifold is a curve $\gamma \in \mathcal{G}[J, X](J \subseteq \mathbb{R})$ satisfying $\gamma^{\prime \prime}=0$.

By (5.2) $\gamma$ is a geodesic in $(X, \hat{g})$ if and only if the usual local formula holds, i.e., if and only if

$$
\left[\left(\frac{d^{2} \gamma_{\varepsilon}^{k}}{d t^{2}}+\sum_{i, j} \hat{\Gamma}_{\varepsilon i j}^{k} \frac{\gamma_{\varepsilon}^{i}}{d t} \frac{\gamma_{\varepsilon}^{j}}{d t}\right)_{\varepsilon}\right]=0 \quad \text { in } \mathfrak{X}_{\mathcal{G}}(\gamma)
$$

\section{Applications to General Relativity}

Before introducing the generalized curvature tensor and its contractions entering Einstein's equations we briefly comment on the shortcomings of classical methods and, in particular, of the linear distributional geometry (as introduced e.g., in 30], 34]) especially in the context of general relativity. While successfully used within linear field theories (e.g., point charges in electrodynamics), applications of distributional methods to general relativistic problems have been rare in the literature; the source of all difficulties of course is the nonlinearity of the field equations.

On the one hand, in the context of (local) existence and uniqueness theorems for Einstein's equations, one is bound to work with Sobolev spaces of high enough regularity so that the metric belongs to some classical function algebra. Roughly speaking, the classical local existence theorems guarantee the existence of a unique solution $g$ to the equations (formulated as an initial value problem along a spacelike hypersurface $\Sigma$ for a Riemannian metric $h$ and its extrinsic curvature $K$ satisfying the constraint equations) with the metric $\left.g \in \mathcal{C}^{0}\left([0, T) ; H^{s}(\Sigma)\right)\right) \cap \mathcal{C}^{1}\left([0, T) ; H^{s-1}(\Sigma)\right)$ for data $h, K$ chosen in $H^{s-1}(\Sigma)$ provided that $s>4$ (cf. [17, 36]). In fact, the optimal local existence result in the case of asymptotic flatness actually only requires $s>5 / 2$ and recent developments by Klainerman and Rodnianski [18] aim at further improving this to the bound $s>2$.

On the other hand, when dealing with special (constructive) solutions of Einstein's equations, one has to cope with the problem of computing the curvature of a given metric of low differentiability (in particular, distributional) which again is problematic due to the nonlinearities involved. Within the $\mathcal{D}^{\prime}$-framework it is nevertheless possible to consistently describe sources of the gravitational field concentrated (i.e., the energy-momentum tensor supported) on a submanifold of codimension one in spacetime (so-called thin shells, cf. [15]). However, in a classical paper [1] Geroch and Traschen have shown that within classical (linear) distribution theory gravitating sources confined to a submanifold of codimension greater than one in spacetime (hence, in particular, such interesting objects as cosmic strings) are excluded from a mathematically rigorous and at the same time physically sensible description. By the latter we mean the existence of an appropriate notion of convergence of metrics which ensures the convergence of the respective curvature tensors.

Here we are going to introduce a setting that is primarily intended to cope with the latter situation described above which at the same time is mathematically rigorous and physically sensible. 
We start by defining the generalized Riemann, Ricci, scalar and Einstein curvatures from an invariant point of view. It is then clear that all the classical formulae will hold on the level of representatives, i.e., all the symmetry properties of the respective classical tensor fields carry over to the new setting. Moreover, the Bianchi identities hold in the generalized sense.

Definition 6.1. Let $(X, \hat{g})$ be a generalized pseudo-Riemannian manifold with Levi-Civita connection $\hat{D}$.

(i) The generalized Riemannian curvature tensor $\hat{R}_{a b c}{ }^{d} \in \mathcal{G}_{3}^{1}(X)$ is defined by

$$
\hat{R}_{\xi, \eta} \zeta:=\hat{D}_{[\xi, \eta]} \zeta-\left[\hat{D}_{\xi}, \hat{D}_{\eta}\right] \zeta .
$$

(ii) We define the generalized Ricci curvature tensor $\hat{R}_{a b} \in \mathcal{G}_{2}^{0}(X)$ by the usual contraction of the generalized Riemann tensor

$$
\hat{R}_{a b}:=\hat{R}_{c a b}{ }^{c} .
$$

(iii) The generalized curvature scalar (or Ricci scalar) $\hat{R} \in \mathcal{G}(X)$ is defined by the usual contraction of the generalized Ricci tensor

$$
\hat{R}:=\hat{g}^{a b} \hat{R}_{a b} .
$$

(iv) Finally, we define the generalized Einstein tensor $\hat{G}_{a b} \in \mathcal{G}_{2}^{0}(X)$ by

$$
\hat{G}_{a b}:=\hat{R}_{a b}-\frac{1}{2} \hat{R} \hat{g}_{a b} .
$$

The framework developed above opens the gate to a wide range of applications in general relativity. Definition 3.4 is capable of modelling a large class of singular spacetimes while at the same time its (generalized) curvature quantities simply may be calculated by the usual coordinate formulae. Hence we are in a position to mathematically rigorously formulate Einstein's equations for generalized metrics. Moreover, we have at our disposal several theorems (which essentially are rooted in [24, Prop. 3) guaranteeing consistency with respect to linear distributional geometry resp. the smooth setting.

Theorem 6.2. Let $(X, \hat{g})$ be a generalized pseudo-Riemannian manifold with $\hat{g}_{a b}$ $\approx_{k} g_{a b}$. Then all the generalized curvature quantities defined above are $\mathcal{C}^{k-2}$ associated with their classical counterparts.

In particular, if a generalized metric $\hat{g}_{a b}$ is $\mathcal{C}^{2}$-associated to a vacuum solution of Einstein's equations, then we have for the generalized Ricci tensor,

$$
\hat{R}_{a b} \approx_{0} R_{a b}=0 .
$$

Hence $\hat{R}_{a b}$ satisfies the vacuum Einstein equations in the sense of 0 -association (cf. the remarks after Prop. 18 in the revised version of 41).

Generally speaking, whenever we encounter a spacetime metric of low differentiability in general relativity, we may proceed along the lines of the following blueprint to obtain a mathematically and physically satisfactory description of the singular spacetime geometry: first we have to transfer the classically singular metric to a generalized one. This may be done by some "canonical" smoothing or by some other physically motivated regularization (see also the remarks on nonlinear modelling preceding Def. 2 in [24]). Once the generalized setting has been entered, all curvature quantities may be calculated simply by using componentwise classical calculus. All classical concepts literally carry over to the new framework and one may treat, e.g., the Ricci tensor, geodesics, geodesic deviation, etc. within this 
nonlinear distributional geometry. Finally, one may use the concept of association to return to the distributional or $\mathcal{C}^{k}$-level for the purpose of interpretation.

This program has been carried out for a conical metric (representing a cosmic string) by Clarke, Vickers and Wilson (see [6], [42], [43] for a treatment in the full setting of Colombeau's construction) rigorously assigning to it a distributional curvature and (via the field equations) the heuristically expected energy-momentum tensor. Moreover, the nonlinear generalized function setting was used in [2], 3] to calculate the energy momentum tensor of the extended Kerr geometry as well as in 14 to unify several distributional approaches to the Schwarzschild geometry. Finally, a complete distributional description of impulsive pp-wave spacetimes was achieved in 39], 22, 23].

An in-depth analysis of the interrelations between the setting introduced here and the one of Geroch and Traschen ([11]) as well as a study of spherical impulsive gravitational waves in this framework is the subject of ongoing research.

We close this work by discussing the geodesic equation of impulsive pp-waves in the present setting. In [39, 22, 23] the geometry of an impulsive pp-wave was described by the following generalized line element (cf. 3.7 (iii)):

$$
\hat{d s}^{2}=f(x, y) D(u) d u^{2}-d u d v+d x^{2}+d y^{2} .
$$

Here the spacetime manifold $X$ is taken to be $\mathbb{R}^{4}$ and $D$ is a generalized delta function, i.e., $D=\operatorname{cl}\left[\left(\delta_{\varepsilon}\right)_{\varepsilon}\right]$, with $\int \delta_{\varepsilon} \rightarrow 1$ the support of $\delta_{\varepsilon}$ shrinking to 0 and $\delta_{\varepsilon}$ uniformly bounded in $L^{1}$ for small $\varepsilon$ (cf. [31], Def. 7.1). Physically this spacetime describes a gravitational impulse located at the null-hypersurface $u=0$ in Minkowski space; the curvature vanishes everywhere but on the impulse.

Equation (6.1) provides an example of a general regularization procedure suggested by the physical situation to be modelled (cf. the discussion in Section 1). In fact, generalized delta functions in the above sense provide a very general class of regularizations of delta-type singularities. Also, (6.1) complies with viewing the singular metric itself as an impulsive limit of sandwich waves of infinitely short duration.

We have to solve the system (5.3) for the metric (6.1). Due to the special form of the metric, the first equation (i.e., $k=0$ ) is trivial, so that (using coordinates $u, v$ and $x^{i}=(x, y)$ as in (6.1) $u$ may be used as an affine parameter along the geodesics and system (5.3) reduces to

$$
\begin{aligned}
\ddot{v}_{\varepsilon}(u) & =f\left(x_{\varepsilon}^{j}(u)\right) \dot{\delta}_{\varepsilon}(u)+2 \partial_{i} f\left(x_{\varepsilon}^{j}(u)\right) \dot{x}_{\varepsilon}^{i}(u) \delta_{\varepsilon}(u), \\
\ddot{x}_{\varepsilon}^{i}(u) & =\frac{1}{2} \partial_{i} f\left(x_{\varepsilon}^{j}(u)\right) \delta_{\varepsilon}(u) .
\end{aligned}
$$

This system of nonlinear ODEs was shown to be uniquely solvable in $\mathcal{G}(\mathbb{R})^{3}$ (cf. [22], Th. 1) for given initial conditions $\left(v_{\varepsilon}, x_{\varepsilon}^{i}\right)(-1)=\left(v_{0}, x_{0}^{i}\right)$ and $\left(\dot{v}_{\varepsilon}, \dot{x}_{\varepsilon}^{i}\right)(-1)=\left(\dot{v}_{0}, \dot{x}_{0}^{i}\right)$. The resulting geodesic is an element of $\mathcal{G}[\mathbb{R}, X]$. Moreover, the generalized solution (by [22], Th. 3) possesses the following distributional shadow:

$$
\begin{aligned}
x^{i} \approx & x_{0}^{i}+\dot{x}_{0}^{i}(1+u)+\frac{1}{2} \partial_{i} f\left(x_{0}^{j}+\dot{x}_{0}^{j}\right) u_{+}, \\
v \approx & v_{0}+\dot{v}_{0}(1+u)+f\left(x_{0}^{j}+\dot{x}_{0}^{j}\right) H(u) \\
& +\partial_{i} f\left(x_{0}^{j}+\dot{x}_{0}^{i}\right)\left(\dot{x}_{0}^{i}+\frac{1}{4} \partial_{i} f\left(x_{0}^{j}+\dot{x}_{0}^{j}\right)\right) u_{+},
\end{aligned}
$$

where $H$ denotes the Heaviside function and $u_{+}=H(u) u$. Hence the macroscopic aspect of the generalized geodesics displays the physically sensible behavior of the 
geodesics being refracted broken straight lines. It should be noted, though, that the explicit calculation of distributional limits in fact is based on the fact that the underlying manifold is given as $\mathbb{R}^{4}$ since in linear distribution theory (contrary to the Colombeau setting) there is no concept of generalized functions valued in a manifold.

Finally, we would like to emphasize that the framework developed in the previous sections for the first time allows a comprehensive and consistent interpretation of the calculations given, e.g., in [6], [14], 22], 23].

\section{ACKNOWLEDGMENTS}

We would like to thank Michael Grosser and Michael Oberguggenberger for numerous discussions that importantly contributed to the final form of the manuscript. Also, we are indebted to the referees for several helpful comments.

\section{REFERENCES}

[1] Aragona, J., Juriaans, S. O. Some structural properties of the topological ring of Colombeau's generalized numbers. Comm. Algebra, 29 (5), 2201-2230, 2001.

[2] Balasin, H. Distributional energy-momentum tensor of the extended Kerr geometry. Class. Quant. Grav., pages 3353-3362, 1997. MR 99f:83009

[3] Balasin, H. Distributional aspects of general relativity: The example of the energy-momentum tensor of the extended Kerr geometry. In Grosser, M., Hörmann, G., Kunzinger, M., Oberguggenberger, M., editor, Nonlinear Theory of Generalized Functions, volume 401 of CRC Research Notes, pages 231-239, Boca Raton, 1999. CRC Press. MR 2000g:83008

[4] Bhatia, R. Perturbation Bounds for Matrix Eigenvalues, volume $\mathbf{1 6 2}$ of Pitman Research Notes in Mathematics. Longman, Harlow, U.K., 1987. MR 88k:15020

[5] Bourbaki, N. Algebra I, Chapters 1-3. Elements of Mathematics. Hermann, Paris, and Addison-Wesley, Massachusetts, 1974. MR 50:6689

[6] Clarke, C. J. S., Vickers, J. A., Wilson, J. P. Generalised functions and distributional curvature of cosmic strings. Class. Quant. Grav., 13, 1996. MR 97g:83085

[7] Colombeau, J. F. New Generalized Functions and Multiplication of Distributions. North Holland, Amsterdam, 1984. MR 86c: 46042

[8] Colombeau, J. F. Elementary Introduction to New Generalized Functions. North Holland, Amsterdam, 1985. MR 87f:46064

[9] Dapić, N., Kunzinger, M., Pilipović, S. Symmetry group analysis of weak solutions. Proc. London Math. Soc., 84(3):686-710, 2002. CMP 2002:09

[10] De Roever, J. W., Damsma, M. Colombeau algebras on a $\mathcal{C}^{\infty}$-manifold. Indag. Mathem., N.S., 2(3), 1991. MR 93e:46046

[11] Geroch, R., Traschen, J. Strings and other distributional sources in general relativity. Phys. Rev. D, 36(4):1017-1031, 1987. MR 89d:83048

[12] Grosser, M., Farkas, E., Kunzinger, M., Steinbauer, R. On the foundations of nonlinear generalized functions I, II. Mem. Amer. Math. Soc. 153, 2001.

[13] Grosser, M., Kunzinger, M., Steinbauer, R., Vickers, J. A global theory of algebras of generalized functions. Adv. Math., 166(1):50-72, 2002. CMP 2002:09

[14] Heinzle, J. M., Steinbauer, R. Remarks on the distributional Schwarzschild geometry. J. Math. Phys., 43(3):1493-1508, 2002.

[15] Israel, W. Singular hypersurfaces and thin shells in general relativity. Nuovo Cim., 44B(1):1$14,1966$.

[16] Kamleh, W. Signature changing space-times and the new generalised functions. Preprint, gr-qc/0004057, 2000.

[17] Klainerman, S., Nicoló, F. On local and global aspects of the Cauchy problem in general relativity. Class. Quant. Grav., 16:R73-R157, 1999. MR 2000h:83006

[18] Klainerman, S., Rodnianski, I. Rough solutions of the Einstein-vacuum equations. Preprint, math.AP/0109173, 2001.

[19] Kriegl, A., Michor, P. W. The Convenient Setting of Global Analysis, volume $\mathbf{5 3}$ of Math. Surveys Monogr. Amer. Math. Soc., Providence, RI, 1997. MR 98i:58015 
[20] Kunzinger, M. Generalized functions valued in a smooth manifold. Monatsh. Math., to appear (available electronically at http://arxiv.org/abs/math.FA/ 0107051), 2002.

[21] Kunzinger, M., Oberguggenberger, M. Group analysis of differential equations and generalized functions. SIAM J. Math. Anal., 31(6):1192-1213, 2000. MR 2001c:35010

[22] Kunzinger, M., Steinbauer, R. A rigorous solution concept for geodesic and geodesic deviation equations in impulsive gravitational waves. J. Math. Phys., 40:1479-1489, 1999. MR 2001a:83018

[23] Kunzinger, M., Steinbauer, R. A note on the Penrose junction conditions. Class. Quant. Grav., 16:1255-1264, 1999. MR 2000c:83023

[24] Kunzinger, M., Steinbauer, R. Foundations of a nonlinear distributional geometry. Acta Appl. Math., 71, 179-206, 2002.

[25] Mallios, A. Geometry of vector sheaves. Vols. I, II. Mathematics and its Applications, 439. Kluwer Academic Publishers, Dordrecht, 1998. MR 99g:58002 MR 99g:58003

[26] Mallios, A., Rosinger, E. E. Abstract differential geometry, differential algebras of generalized functions, and de Rham cohomology. Acta Appl. Math., 55(3):231-250, 1999. MR 2000e: 58003

[27] Mallios, A., Rosinger, E. E. Space-time foam dense singularities and de Rham cohomology. Acta Appl. Math., 67: 59-89, 2001. MR 2002e:58004

[28] Ligęza, J., Tvrdy, M. On systems of linear algebraic equations in the Colombeau algebra. Math. Bohem., 124(1):1-14, 1999. MR 2000c:46079

[29] Mansouri, R., Nozari, K. A new distributional approach to signature change. Gen. Relativity Gravitation, 32(2):235-269, 2000. MR 2001f:83136

[30] Marsden, J. E. Generalized Hamiltonian mechanics. Arch. Rat. Mech. Anal., 28(4):323-361, 1968. MR 37:534

[31] Oberguggenberger, M. Multiplication of Distributions and Applications to Partial Differential Equations, volume 259 of Pitman Research Notes in Mathematics. Longman, Harlow, 1992. MR 94d:46044

[32] Oberguggenberger, M., Kunzinger, M. Characterization of Colombeau generalized functions by their point values. Math. Nachr., 203:147-157, 1999. MR 2000d:46053

[33] O'Neill, B. Semi-Riemannian Geometry (with Applications to Relativity). Academic Press, New York, 1983. MR 85f:53002

[34] Parker, P. Distributional geometry. J. Math. Phys., 20(7):1423-1426, 1979. MR 81f:83030

[35] Penrose, R., Rindler, W. Spinors and space-time I. Cambridge University Press, 1984. MR 86h:83002

[36] Rendall, A. Local and Global Existence Theorems for the Einstein Equations. Living Rev. Relativity, 3(1). [Online Article]: cited on 2002-01-20, http://www. livingreviews.org/Articles/Volume3/2000-1rendall/. MR 2000i:58053

[37] Simanca, S. R. Pseudo-differential Operators, volume 236 of Pitman Research in Notes in Mathematics. Longman, Harlow, 1990. MR 91i:35222

[38] Steinbauer, R. The ultrarelativistic Reissner-Nordstrøm field in the Colombeau algebra. J. Math. Phys., 38:1614-1622, 1997. MR 98f:83017

[39] Steinbauer, R. Geodesics and geodesic deviation for impulsive gravitational waves. J. Math. Phys., 39:2201-2212, 1998. MR 99a:83027

[40] Vickers, J. A. Nonlinear generalized functions in general relativity. In Grosser, M., Hörmann, G., Kunzinger, M., Oberguggenberger, M., editor, Nonlinear Theory of Generalized Functions, volume 401 of CRC Research Notes, pages 275-290, Boca Raton, 1999. CRC Press. MR 2000g:83056

[41] Vickers, J., Wilson, J. A nonlinear theory of tensor distributions. ESI-Preprint (available electronically at http://www.esi.ac.at/ESI-Preprints.html), 566, 1998.

[42] Vickers, J. A., Wilson, J. P. Invariance of the distributional curvature of the cone under smooth diffeomorphisms. Class. Quantum. Grav., 16:579-588, 1999. MR 2000a:83096

[43] Wilson, J. P. Distributional curvature of time dependent cosmic strings. Class. Quantum Grav., 14:3337-3351, 1997. MR 99e:83018 
Department of Mathematics, University of Vienna, Strudlhofg. 4, A-1090 Wien, Austria

E-mail address: Michael.Kunzinger@univie.ac.at

Department of Mathematics, University of Vienna, Strudlhofg. 4, A-1090 Wien, AUstria

E-mail address: roland.steinbauer@univie.ac.at 